\title{
Meta-model of a large credit risk portfolio in the Gaussian copula model
}

\author{
F. Bourgey*; $\quad$ E. Gobet $\dagger \quad$ C. Rey ${ }^{\ddagger}$ \\ Current version: October 8, 2019
}

\begin{abstract}
We design a meta-model for the loss distribution of a large credit portfolio in the Gaussian copula model. Using both the Wiener chaos expansion on the systemic economic factor and a Gaussian approximation on the associated truncated loss, we significantly reduce the computational time needed for sampling the loss and therefore estimating risk measures on the loss distribution. The accuracy of our method is confirmed by many numerical examples.
\end{abstract}

KEYwords: Monte Carlo simulation; portfolio credit risk; polynomial chaos expansion; meta-model

MSC2010: 91G60; 91G40; 33C45; 41A10

\section{Introduction}

In credit risk modeling, one needs to properly model and sample the loss distribution $\mathcal{L}$ associated to the credit portfolio of $K$ obligors in order to compute the probability of large losses $\mathbb{P}(\mathcal{L}>x)$, for large $x$. More precisely, the loss $\mathcal{L}$ takes the form :

$$
\mathcal{L}:=\sum_{k=1}^{K} l_{k} Y_{k}
$$

where $Y_{k}=1$ or 0 according to the default (or not) of the $k$ th obligor and $l_{k}$ is the related Loss Given Default. Such quantity appears in many credit risk measurements such as Var, CVar... (see [ADEH99, CLV04, MFE05, BR13] for extensive references on risk measures and credit risk). The problem we address in this work corresponds to a large number $K$ of obligors: indeed, it is often that banks are facing portfolio with $K \geq 10^{5}$ obligors. Therefore, any Monte-Carlo (MC) simulation scheme is hugely time-consuming, since generating a single sample of $\mathcal{L}$ requires to compute all terms of the sum (1.1). Estimating $\mathbb{P}(\mathcal{L}>x)$ by $\mathrm{MC}$ can then take hours using only few hundreds/thousands samples, whence resulting in a quite imprecise estimation of the probability of interest. To overcome this, we design a new meta-model for sampling the loss, based on the Wiener chaos decomposition. A meta-model is an approximation of the true model, which has the advantage of being much faster to evaluate, and consequently, which is more suitable for massive MC simulations and asymptotic statistical error analysis. As we will see, the level of approximation of $\mathcal{L}$ can be tuned as the user wants, so that we obtain a full range of meta-models from exact to rough, with decreasing accuracy but increasing speed-up.

\footnotetext{
*Centre de Mathématiques Appliquées (CMAP), CNRS, Ecole Polytechnique, Institut Polytechnique de Paris, Route de Saclay, 91128 Palaiseau Cedex, France. Email: florian.bourgey@polytechnique.edu

${ }^{\dagger}$ CMAP, Ecole Polytechnique. Email: emmanuel.gobet@polytechnique.edu

${ }^{\ddagger}$ CMAP, Ecole Polytechnique. Email: clement.rey@polytechnique.edu.

This research benefited from the support of the Chair Stress Test, RISK Management and Financial Steering, led by the French Ecole polytechnique and its Foundation and sponsored by BNP Paribas. We thank the STFS team of BNP Paribas for fruitful discussions and constructive feedbacks.
} 
Our contributions. We derive the meta-model in the framework where the default variables $\left(Y_{k}: k\right)$ obey a copula model, first introduced by Li [Li00] to cop with the credit dependency analysis. See also [ASB03], where the one-factor normal copula model is used for the CDO (Collateralized Debt Obligation) pricing. See Section 2 for a precise description of the model. All the defaults depend on a common variable $Z$ (the systemic risk factor, which plays the role of a macro-economic variable), modelled as standard normal random variables, and conditionally to $Z$, defaults are independent. Extensions to multifactor is discussed in details in Subsection 3.3.

Our main result is a meta-model for $\mathcal{L}$ which takes the following form (see Theorem 7 ):

$$
\mathcal{L}(\omega) \approx \sum_{i=0}^{I} \varepsilon_{K, i}(\omega) \operatorname{He}_{i}(Z(\omega))
$$

where

- $\left(\operatorname{He}_{i}(\cdot): i \geq 0\right)$ is the family of probabilists' Hermite polynomials;

- I a truncation parameter tuning the accuracy of the meta-model;

- the (random) coefficients $\varepsilon_{K, i}$ form a normal vector with explicit characteristics (depending on the LGD and other default parameters), and independent of $Z$. The significant gain is that these characteristics can be computed off-line, so that sampling the meta-model reduces to sample a $I$-dimensional normal vector and the systemic risk factor $Z$. Therefore, instead of sampling $K$ terms, we essentially sample $I$ terms.

The meta-model (1.2) writes as a (truncated) Wiener chaos decomposition of $\mathcal{L}$ in the $Z$ variable.

Background results. At the beginning of the 2000's, there have been numerous works related to the numerical approximation of CDO pricing (before the 2007-crisis, these products were very popular). It is related to the computation of the tranche function $\mathbb{E}\left[(\mathcal{L}-x)_{+}\right]$for many attachement levels $x$ and for some loss $\mathcal{L}$ of the form (1.1). The main methodology is to derive explicit approximations for conditional quantities $\mathbb{E}\left[(\mathcal{L}-x)_{+} \mid Z\right]$ (and then integrate over $\left.Z^{1}\right)$ : the problem is simpler since the conditional loss writes as a sum of independent random variables. For instance, in [Vas91], Vasicek proposed a Gaussian approximation using the principle of Central Limit Theorem. The authors of [JEK08] have significantly improved the zero-order Gaussian approximation by identifying some correction terms using the Stein method; alternatively to Gaussian approximations available for some specific parameter regime, they also provide Poisson approximations. Still taking advantage of the sum of independent random variables, some works made use of the explicit characteristic functions available for the conditional loss and the use of saddlepoint techniques to provide approximations of the tranche function, see [YHZ06] for instance. All these references (and others therein) provide approximations of the conditional loss distribution using a probability distribution with parameters $\theta$ depending on $Z$. For large portfolio $(K \rightarrow+\infty)$, this may be computationally very intensive, since for any sampled risk factor $Z$ (a priori many for computing the outer non-conditional expectation), the parameters $\theta(Z)$ have to be re-evaluated hence recomputing all the $K$ terms in (1.1) conditionally to $Z$. Our approach based on the Wiener chaos decomposition is different since we factorize the dependence with respect to (w.r.t.) $Z$ from the other variables (see Theorem 7), so that the critical part (related to $K$ large) can be made off-line.

A second difference between the above references and our work is that we derive directly an approximation on $\mathcal{L}$, independently of the threshold $x$ appearing in the tranche function $\mathbb{E}\left[(\mathcal{L}-x)_{+}\right]$or in the survival function $\mathbb{P}(\mathcal{L}>x)$. We are working directly at the level of approximating the loss distribution, rather than its statistics (through test functions): our approach is closer to a simulation-based experience that a risk manager could wish to have (with visualisation of loss histograms ...).

\footnotetext{
${ }^{1}$ which is standard using Monte Carlo method or any numerical integration method
} 
Another family of numerical methods to sample $\mathcal{L}$ and efficiently compute their statistics is the MC method with importance sampling (IS). This is quite efficient, especially for extreme loss thresholds $x$. Important references are [GL05], where the authors apply IS conditionally on $Z$ (the common factors affecting obligors) and IS to the factors themselves. The tuning of this approach depends on $x$ and the computational time also suffers from having many obligors $(K \rightarrow+\infty)$. Our work takes a different route as we wish to approximate the full distribution of $\mathcal{L}$, especially as $K \rightarrow+\infty$.

Although Gaussian meta-modeling is quite well-known among the Uncertainty Quantification community, we are not aware of any use for financial engineering problems, and in particular for assessing portfolio credit risk. For a recent account on meta-modeling with polynomial chaos and Gaussian processes, see [LMS16].

Organisation of the paper. In section 2, we briefly recall the Gaussian copula model of [Li00], state known properties on probabilists' Hermite polynomials and the Wiener chaos decomposition. Then, we compute the chaos decomposition of the indicator function $\mathbb{1}_{c \leq Z}$ where $Z$ is standard normal and $c$ a constant, and apply this to obtain a stochastic chaos decomposition of order $I$ (denoted $\mathcal{L}_{I}$ ) for the portfolio loss $\mathcal{L}$. We then show how the coefficients of this decomposition can be well approximated by suitable multidimensional normal distributions. We provide also the $L_{2}$ error and a central limit theorem for the approximation $\mathcal{L}_{I}$. Technicalities are postponed to Section 5. In Section 3, we give explicit expressions to compute the characteristics of the normal vector needed for the approximation and test our approach on different portfolio scenarios. We summarize our main contributions and conclude in Section 4.

\section{Notations.}

- $\mathbb{N}=\{0,1, \ldots$,$\} for the non-negative integers.$

- Let $n \in \mathbb{N}^{*}, x \in \mathbb{R}^{n}, \Sigma \in \mathbb{R}^{n \times n}$ positive definite and $X \stackrel{d}{=} \mathcal{N}(0, \Sigma)$, set

$$
\Phi_{\Sigma}(x):=\mathbb{P}(X \leq x)=\int_{-\infty}^{x_{1}} \cdots \int_{-\infty}^{x_{n}} \frac{e^{-\frac{1}{2} t^{\top} \Sigma^{-1} t}}{(2 \pi)^{\frac{n}{2}}(\operatorname{det}(\Sigma))^{\frac{1}{2}}} \mathrm{~d} t_{1} \ldots \mathrm{d} t_{n} .
$$

In the case $n=1$ and $\Sigma=1$, we simply write $\Phi$ for $\Phi_{\Sigma}$.

- For $n, m \in \mathbb{N}, \delta_{n m}$ denotes the Kronecker delta i.e $\delta_{n m}=1$ if $n=m$ and 0 otherwise.

\section{Gaussian Copula Model, Wiener chaos decomposition and main result on meta-models}

\subsection{Gaussian copula model for the portfolio credit risk problem}

We are considering the one-year loss of a large portfolio composed of $K$ obligors, each having a deterministic LGD $l_{k} \geq 0$. The marginal default probability of the $k$ th obligor is denoted by $p_{k}$, it is assumed to be known either from credit ratings, market prices, corporate bonds or credit default swaps. In this framework, the one-year total loss from defaults (without any provision) is defined as :

$$
\mathcal{L}:=\sum_{k=1}^{K} l_{k} Y_{k},
$$

where $Y_{k}$ represents the default indicator for the $k$ th obligor at one-year; i.e $Y_{k}=1$ if the $k$ th obligor defaults or 0 otherwise. We assume that the defaults obey to the copula model. Namely, in the Gaussian copula model,

$$
Y_{k}=\mathbb{1}_{X_{k} \geq c_{k}}
$$


with stochastic factors $X_{k}$ and thresholds $c_{k}$ (referred as a default boundary), and the dependence among obligors is achieved through a multivariate normal vector $\left(X_{1}, \ldots, X_{K}\right)$ with standardized entries $\left(X_{k} \stackrel{d}{=}\right.$ $\mathcal{N}(0,1))$.

For the sake of clarity, we choose to first focus our analysis on a 1-dimensional factor model where $X_{k}$ takes the form :

$$
X_{k}=\rho_{k} Z+\sqrt{1-\rho_{k}^{2}} \varepsilon_{k}
$$

where $Z \stackrel{d}{=} \mathcal{N}(0,1)$ is a systemic risk factor, $\varepsilon_{k} \stackrel{d}{=} \mathcal{N}(0,1)$ is an idiosyncratic risk associated with the $k$ th obligor (independent from $Z), \rho_{k} \in(-1,1)$ a correlation parameter. The $\left(\varepsilon_{k}\right)_{1 \leq k \leq K}$ are independent, and independent from $Z$. The extension to a $d$-multi-factor model is presented in Section 3.3.

We assume from now on that $\rho_{k} \neq 0$ : indeed, from the economic point of view, there is always a dependency between the default of an obligor and the systemic risk factor. From the mathematical point of view, the set of random variables $Y_{k}$ associated to $\rho_{k}=0$ forms an independent sequence, and independent from the other $Y_{l}$, their marginal distributions are just Bernoulli distributions, thus they can be easily treated separately from the dependent $Y_{l}$.

The default boundary $c_{k}:=\Phi^{-1}\left(1-p_{k}\right)=-\Phi^{-1}\left(p_{k}\right)$ is such that it matches the constraint $p_{k}=$ $\mathbb{P}\left(Y_{k}=1\right)$ : indeed, the choice of $c_{k}$ readily implies that

$$
\mathbb{P}\left(Y_{k}=1\right)=\mathbb{P}\left(X_{k} \geq c_{k}\right)=\Phi\left(-c_{k}\right)=p_{k} .
$$

Then, the default event writes (remembering that we exclude the case $\rho_{k}=0$ ) :

$$
\left\{X_{k} \geq c_{k}\right\}=\left\{a_{k} \varepsilon_{k}+b_{k} \leq \frac{\rho_{k}}{\left|\rho_{k}\right|} Z\right\} \quad \text { where } a_{k}=\frac{-\sqrt{1-\rho_{k}^{2}}}{\left|\rho_{k}\right|}, b_{k}=\frac{-\Phi^{-1}\left(p_{k}\right)}{\left|\rho_{k}\right|} .
$$

Here, we emphazise that both random variables are normally distributed :

$$
\frac{\rho_{k}}{\left|\rho_{k}\right|} Z \stackrel{d}{=} \mathcal{N}(0,1), \quad a_{k} \varepsilon_{k}+b_{k} \stackrel{d}{=} \mathcal{N}\left(b_{k}, a_{k}^{2}\right)
$$

\subsection{Wiener chaos decomposition of the indicator function}

We first recall some well-known results on the probabilists' Hermite polynomials of degree $i \in \mathbb{N}$. See [Sze75, Chapter V] and [Fun92] for extensive references on orthogonal polynomials. For (2.4)-(2.5)-(2.7)-(2.13), we refer to [LK10, Section B.1.2]. For (2.11) and (2.12), see [Nua06, Chapter 1] and [Bai48] respectively. Equalities (2.6) and (2.9) readily follow from (2.7). Equality (2.8) is derived from the exact Taylor expansion w.r.t. $x$ at point $y$, combined with $\operatorname{He}_{i}^{(m)}(y)=\frac{i !}{(i-m) !} \mathrm{He}_{m}(y)(0 \leq m \leq i)$ which follows from (2.11). Equality (2.10) is a direct consequence of (2.5) and (2.11). The exponential generating function (2.14) is obtained from the series expansion of $e^{x w-\frac{w^{2}}{2}}=\sum_{i \geq 0} \frac{c_{i}}{i !} w^{i}$ with $c_{i}=\left.\frac{\partial^{i}}{\partial w^{i}}\left(e^{x w-\frac{w^{2}}{2}}\right)\right|_{w=0}=\operatorname{He}_{i}(x)$.

Proposition 1. For every $i \in \mathbb{N}$, the following properties hold for the probabilists' Hermite polynomials :

$$
\begin{aligned}
& \mathrm{He}_{i}(x)=(-1)^{i} e^{\frac{x^{2}}{2}} \frac{\mathrm{d}^{i}}{\mathrm{~d} x^{i}}\left(e^{-\frac{x^{2}}{2}}\right), \\
& \mathrm{He}_{0}(x)=1, \quad \operatorname{He}_{1}(x)=x, \quad \mathrm{He}_{i+2}(x)=x \mathrm{He}_{i+1}(x)-(i+1) \mathrm{He}_{i}(x), \\
& \mathrm{He}_{i}(-x)=(-1)^{i} \mathrm{He}_{i}(x), \\
& \mathrm{He}_{i}(x)=i ! \sum_{m=0}^{\left\lfloor\frac{i}{2}\right\rfloor} \frac{(-1)^{m}}{m !(i-2 m) !} \frac{x^{i-2 m}}{2^{m}}, \\
& \mathrm{He}_{i}(x+y)=\sum_{m=0}^{i}\left(\begin{array}{c}
i \\
m
\end{array}\right) x^{i-m} \mathrm{He}_{m}(y), \\
& \mathrm{He}_{2 i}(0)=(-1)^{i} \frac{(2 i) !}{2^{i} i !}, \quad \operatorname{He}_{2 i+1}(0)=0,
\end{aligned}
$$




$$
\begin{aligned}
& \mathrm{He}_{i}^{\prime \prime}(x)-x \mathrm{He}_{i}^{\prime}(x)+i \mathrm{He}_{i}(x)=0, \\
& \mathrm{He}_{i+1}^{\prime}(x)-(i+1) \mathrm{He}_{i}(x)=0, \\
& \forall A \in \mathbb{R}, \quad \operatorname{He}_{i}(A x)=\sum_{m=0}^{\left\lfloor\frac{i}{2}\right\rfloor} A^{i-2 m}\left(A^{2}-1\right)^{m} \frac{i !}{m !(i-2 m) !} 2^{-m} \mathrm{He}_{i-2 m}(x), \\
& \forall i, j \in \mathbb{N}, \quad \int_{\mathbb{R}} \operatorname{He}_{i}(x) \mathrm{He}_{j}(x) \frac{e^{-\frac{x^{2}}{2}}}{\sqrt{2 \pi}} \mathrm{d} x=i ! \delta_{i j}, \\
& \forall x, w \in \mathbb{R}, \quad e^{x w-\frac{w^{2}}{2}}=\sum_{i=0}^{\infty} \operatorname{He}_{i}(x) \frac{w^{i}}{i !} .
\end{aligned}
$$

We recall that any measurable function $\varphi: \mathbb{R} \mapsto \mathbb{R}$, such that $\mathbb{E}\left[\varphi^{2}(Z)\right]<+\infty$, can be decomposed into $L_{2}$ (chaos decomposition) as

$$
\varphi(Z)=\sum_{i=0}^{\infty} \alpha_{i} \mathrm{He}_{i}(Z)
$$

with $\alpha_{i}=\mathbb{E}\left[\varphi(Z) \mathrm{He}_{i}(Z)\right] / i$ !. Before applying this result to the indicator function on the event (2.3), we need the following Proposition.

Proposition 2. Let $Z$ be a standard normal random variable and $c \in \mathbb{R}$. Then the following chaos decomposition holds :

$$
\mathbb{1}_{c \leq Z}=\sum_{i=0}^{\infty} \alpha_{i}(c) \mathrm{He}_{i}(Z)
$$

with $\alpha_{0}(c)=\Phi(-c)$ and for $i \in \mathbb{N}^{*}$,

$$
\alpha_{i}(c)=\frac{e^{-\frac{c^{2}}{2}} \mathrm{He}_{i-1}(c)}{i ! \sqrt{2 \pi}}=\frac{e^{-\frac{c^{2}}{2}}}{i \sqrt{2 \pi}} \sum_{m=0}^{\left\lfloor\frac{i-1}{2}\right\rfloor} \frac{(-1)^{m}}{m !(i-1-2 m) !} \frac{c^{i-1-2 m}}{2^{m}} .
$$

Furthermore, the following relations between coefficients hold:

$$
\forall i \in \mathbb{N}, \quad \alpha_{i+2}(c)=\frac{c}{i+2} \alpha_{i+1}(c)-\frac{i}{(i+1)(i+2)} \alpha_{i}(c) .
$$

The above equality (2.15) holds in $L_{2}$, and thus for almost every $Z$. Actually (see the discussion below), the equality holds for $Z \neq c$.

\subsection{Wiener chaos decomposition of the loss}

At this point, in view of $(2.3)$, since $\frac{\rho_{k}}{\left|\rho_{k}\right|} Z \stackrel{d}{=} Z \stackrel{d}{=} \mathcal{N}(0,1)$, we would like to apply (2.15) with $c=a_{k} \varepsilon_{k}+b_{k}$. However since $(2.15)$ holds a.s. (i.e. except on a set of zero measure $\mathcal{E}_{c}$ ) and the $c$ 's are to be chosen as random, one needs to be cautious. Fortunately, the convergence in (2.15) does not hold only for almost every $Z$, but for all $Z \neq c$. This is a consequence of the Uspensky's criterion for pointwise convergence of Hermite series [Usp26]; moreover, whenever needed, the series on the right-hand side of (2.15) converges to the left-hand side uniformly on any compact set of $Z$-values that does not contain $c$. For the special case, $Z=c$, it converges to $\frac{1}{2}$ (Gibbs phenomenon), see [San91, Section IV.10]. It implies that on the complement of the set $\mathcal{E}=\bigcup_{k=1}^{K}\left\{a_{k} \varepsilon_{k}+b_{k}=\frac{\rho_{k}}{\left|\rho_{k}\right|} Z\right\}$, the equality (2.15) holds simultaneously for $c=a_{k} \varepsilon_{k}+b_{k}$ for all $k \in\{1, \ldots, K\}$. Since the sequence $\left(\varepsilon_{k}\right)_{k=1, \ldots, K}$ is independent from $\frac{\rho_{k}}{\left|\rho_{k}\right|} Z$ and the law of $\frac{\rho_{k}}{\left|\rho_{k}\right|} Z$ has not atoms, the set $\mathcal{E}$ has zero probability. It implies that we have a.s.

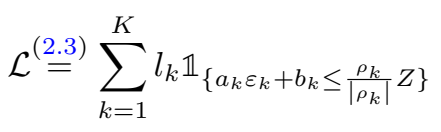




$$
\begin{aligned}
& =\sum_{k=1}^{K} l_{k} \sum_{i=0}^{\infty} \alpha_{i}\left(a_{k} \varepsilon_{k}+b_{k}\right) \operatorname{He}_{i}\left(\frac{\rho_{k}}{\left|\rho_{k}\right|} Z\right) \\
& =\sum_{i=0}^{\infty}\left\{\sum_{k=1}^{K} l_{k} \alpha_{i}\left(a_{k} \varepsilon_{k}+b_{k}\right) \frac{\rho_{k}^{i}}{\left|\rho_{k}\right|^{i}}\right\} \operatorname{He}_{i}(Z) .
\end{aligned}
$$

The last equality can be easily obtained noticing that :

$$
\mathrm{He}_{i}\left(\frac{\rho_{k}}{\left|\rho_{k}\right|} Z\right)=\mathrm{He}_{i}(Z) \mathbb{1}_{\rho_{k}>0}+\mathrm{He}_{i}(-Z) \mathbb{1}_{\rho_{k}<0} \stackrel{(2.6)}{=} \operatorname{He}_{i}(Z) \mathbb{1}_{\rho_{k}>0}+(-1)^{i} \mathrm{He}_{i}(Z) \mathbb{1}_{\rho_{k}<0}=\frac{\rho_{k}^{i}}{\left|\rho_{k}\right|^{i}} \operatorname{He}_{i}(Z) .
$$

Truncating the sum over $i$ at $I$ gives the so-called $I$-chaos decomposition of the loss. This is our first main result.

Theorem 3. Under the assumption that $\left(\varepsilon_{k}\right)_{k=1, \ldots, K}$ is independent from $Z$, the chaos decomposition of the loss $\mathcal{L}$ with respect to $Z$ is given by :

$$
\mathcal{L}=\sum_{i=0}^{\infty}\left\{\sum_{k=1}^{K} l_{k} \alpha_{i}\left(a_{k} \varepsilon_{k}+b_{k}\right) \frac{\rho_{k}^{i}}{\left|\rho_{k}\right|^{i}}\right\} \operatorname{He}_{i}(Z),
$$

where the $\alpha_{i}($.$) 's are given in Proposition 2. Then, the associated truncated chaos decomposition of order$ $I \in \mathbb{N}$ of the loss $\mathcal{L}$ or I-chaos decomposition is defined by :

$$
\mathcal{L}_{I}=\sum_{i=0}^{I}\left\{\sum_{k=1}^{K} l_{k} \alpha_{i}\left(a_{k} \varepsilon_{k}+b_{k}\right) \frac{\rho_{k}^{i}}{\left|\rho_{k}\right|^{i}}\right\} \operatorname{He}_{i}(Z) .
$$

The analysis of the truncation error is made possible by a better understanding on how fast the coefficients $\alpha_{i}$ decrease to 0: it will be handled by the next two results.

Lemma 4. For every $n \in \mathbb{N}$, the successive relative maxima of $x \in \mathbb{R} \rightarrow e^{-\frac{x^{2}}{2}}\left|\operatorname{He}_{n}(x)\right|$ form a decreasing (resp. increasing) sequence for $x \geq 0$ (resp. for $x \leq 0$ ).

Corollary 5. For every $n \in \mathbb{N}$, define $M_{n}:=\max _{x \in \mathbb{R}}\left(\frac{e^{-x^{2}} \mathrm{He}_{n}^{2}(x)}{(n+1) !}\right)$ then :

$$
\begin{aligned}
& M_{n}=\left\{\begin{array}{ll}
\frac{(2 m) !}{(2 m+1)(m !)^{2} 2^{2 m}} & \text { if } n=2 m \\
\frac{e^{-x_{1,2 m+2}^{2} \mathrm{He}_{2 m+1}^{2}\left(x_{1,2 m+1}\right)}}{(2 m+2) !} & \text { if } n=2 m+1
\end{array},\right. \\
& M_{2 m+1}<M_{2 m},
\end{aligned}
$$

where $m \in \mathbb{N}$ and $x_{1,2 m+2}>0$ denotes the first positive zero of $\mathrm{He}_{2 m+2}$.

For the reader interested in estimates on the maximum of weighted Hermite polynomials, see the work of Krasikov [Kra04]. Notice that his estimates are different as he considers physicists' Hermite polynomials $\mathrm{H}_{i}($.$) which are linked to the probabilists' Hermite polynomials through the relation \mathrm{H}_{i}(x)=2^{i / 2} \mathrm{He}_{i}(\sqrt{2} x)$. Yet the upper bound obtained in [Kra04, Theorem 1] is not equivalent to that of our Corollary 5 (even after the above rescaling). Our above upper bound is tight and customized to our setting.

We are now in a position to analyse the truncation error related to the chaos decomposition of Proposition 2 and Theorem 3. We recall that $\left(\varepsilon_{k}\right)_{k=1 \ldots K}$ is independent from $Z$. 


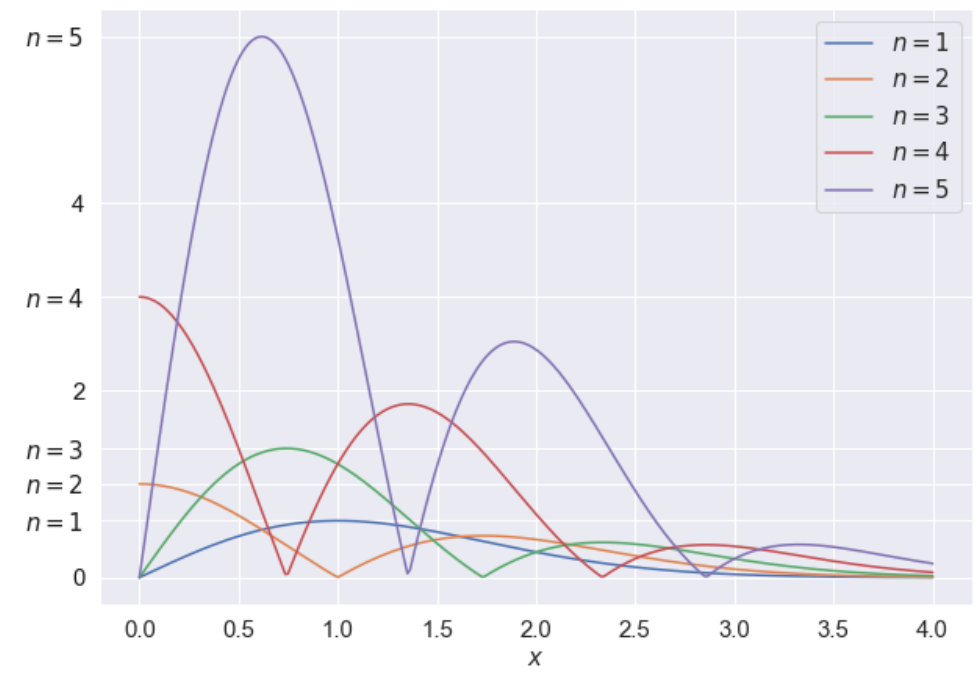

Figure 1: Plot of $x \mapsto e^{-\frac{x^{2}}{2}}\left|\mathrm{He}_{n}(x)\right|$ for $n=1, \ldots, 5$ to illustrate Lemma 4.

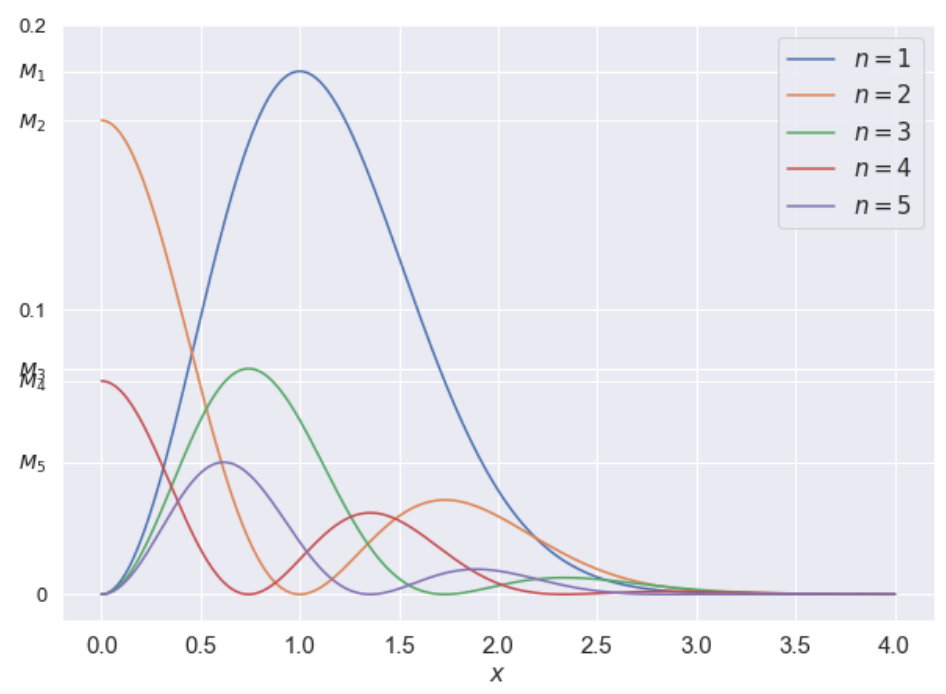

Figure 2: Plot of $x \mapsto \frac{e^{-x^{2}} \mathrm{He}_{n}^{2}(x)}{(n+1) !}$ for $n=1, \ldots, 5$, to illustrate the inequality (2.22).

Theorem 6. Let $I \in \mathbb{N}^{*}, c \in \mathbb{R}$ and $Z$ a standard normal random variable. Then, there exists $C_{(2.23)} \geq 0$ which does not depend on $I$ and $K$, such that:

$$
\sup _{c \in \mathbb{R}} \mathbb{E}\left[\left|\mathbb{1}_{c \leq Z}-\sum_{i=0}^{I} \alpha_{i}(c) \operatorname{He}_{i}(Z)\right|^{2}\right] \leq \frac{C_{(2.23)}}{\sqrt{I}}, \quad \text { and } \quad \mathbb{E}\left[\left|\mathcal{L}-\mathcal{L}_{I}\right|^{2}\right] \leq C_{(2.23)} \frac{\left(\sum_{k=1}^{K} l_{k}\right)^{2}}{\sqrt{I}}
$$

A careful analysis of the proof shows that this result holds without assuming that $\left(\varepsilon_{k}\right)_{k=1 \ldots K}$ forms a independent sequence, only the independence w.r.t. $Z$ is required. It is interesting for the extension to the 
multi-factor model (see the discussion in Section 3.3). According to the numerical experiments reported in Figure 6 , it seems that the above estimates are tight (one can not improve the convergence rate).

Proof of Theorem 6. First note that the second estimate is a direct consequence of the first one. Indeed, starting from (2.18)-(2.19)-(2.21), using positivity of the $l_{k}$ 's, the Cauchy-Schwarz inequality, the fact that $\frac{\rho_{k}}{\left|\rho_{k}\right|} Z \stackrel{d}{=} Z \stackrel{d}{=} \mathcal{N}(0,1)$ and the first estimate, we have :

$$
\begin{aligned}
\mathbb{E}\left[\left|\mathcal{L}-\mathcal{L}_{I}\right|^{2}\right]= & \mathbb{E}\left[\left|\sum_{k=1}^{K} l_{k}\left(\mathbb{1}_{\left\{a_{k} \varepsilon_{k}+b_{k} \leq \frac{\rho_{k}}{\left|\rho_{k}\right|} Z\right\}}-\sum_{i=0}^{I} \alpha_{i}\left(a_{k} \varepsilon_{k}+b_{k}\right) \mathrm{He}_{i}\left(\frac{\rho_{k}}{\left|\rho_{k}\right|} Z\right)\right)\right|^{2}\right] \\
\leq & \sum_{k, k^{\prime}=1}^{K} l_{k} l_{k^{\prime}} \mathbb{E}\left[\left|\mathbb{1}_{\left\{a_{k} \varepsilon_{k}+b_{k} \leq \frac{\rho_{k}}{\left|\rho_{k}\right|} Z\right\}}-\sum_{i=0}^{I} \alpha_{i}\left(a_{k} \varepsilon_{k}+b_{k}\right) \mathrm{He}_{i}\left(\frac{\rho_{k}}{\left|\rho_{k}\right|} Z\right)\right|\right. \\
& \left.\times\left|\mathbb{1}_{\left\{a_{k^{\prime}} \varepsilon_{k^{\prime}}+b_{k^{\prime}} \leq \frac{\rho_{k}}{\left|\rho_{k}\right|} Z\right\}}-\sum_{i=0}^{I} \alpha_{i}\left(a_{k^{\prime}} \varepsilon_{k^{\prime}}+b_{k^{\prime}}\right) \mathrm{He}_{i}\left(\frac{\rho_{k^{\prime}}}{\left|\rho_{k^{\prime}}\right|} Z\right)\right|\right] \\
\leq & \sum_{k, k^{\prime}=1}^{K} l_{k} l_{k^{\prime}} \mathbb{E}\left[\left|\mathbb{1}_{\left\{a_{k} \varepsilon_{k}+b_{k} \leq \frac{\rho_{k}}{\left|\rho_{k}\right|} Z\right\}}-\sum_{i=0}^{I} \alpha_{i}\left(a_{k} \varepsilon_{k}+b_{k}\right) \operatorname{He}_{i}\left(\frac{\rho_{k}}{\left|\rho_{k}\right|} Z\right)\right|^{2}\right]^{1 / 2} \\
& \times \mathbb{E}\left[\left|\mathbb{1}_{\left\{a_{k^{\prime}} \varepsilon_{k^{\prime}}+b_{k^{\prime}} \leq \frac{\rho_{k^{\prime}}}{\left|\rho_{k^{\prime}}\right|} Z\right\}}-\sum_{i=0}^{I} \alpha_{i}\left(a_{k^{\prime}} \varepsilon_{k^{\prime}}+b_{k^{\prime}}\right) \mathrm{He}_{i}\left(\frac{\rho_{k^{\prime}}}{\left|\rho_{k^{\prime}}\right|} Z\right)\right|^{2}\right]^{1 / 2} \\
= & \sum_{k, k^{\prime}=1}^{K} l_{k} l_{k^{\prime}} \mathbb{E}\left[\left|\mathbb{1}_{\left\{a_{k} \varepsilon_{k}+b_{k} \leq Z\right\}}-\sum_{i=0}^{I} \alpha_{i}\left(a_{k} \varepsilon_{k}+b_{k}\right) \mathrm{He}_{i}(Z)\right|^{2}\right]^{1 / 2} \\
& \times \mathbb{E}\left[\left|\mathbb{1}_{\left\{a_{k^{\prime}} \varepsilon_{k^{\prime}}+b_{k^{\prime}} \leq Z\right\}}-\sum_{i=0}^{I} \alpha_{i}\left(a_{k^{\prime}} \varepsilon_{k^{\prime}}+b_{k^{\prime}}\right) \operatorname{He}_{i}(Z)\right|^{1 / 2}\right]^{1 / 2} \\
\leq & \frac{C_{(2.23)}}{\sqrt{I}\left(\sum_{k=1}^{K} l_{k}\right)^{2} .}
\end{aligned}
$$

Now, let us prove the first estimate. The quadratic error of the truncated projection is equal to:

$$
\mathbb{E}\left[\left|\mathbb{1}_{c \leq Z}-\sum_{i=0}^{I} \alpha_{i}(c) \mathrm{He}_{i}(Z)\right|^{2}\right] \stackrel{(2.15)}{=} \mathbb{E}\left[\left|\sum_{i=I+1}^{\infty} \alpha_{i}(c) \mathrm{He}_{i}(Z)\right|^{2}\right] \stackrel{(2.13)}{=} \sum_{i=I+1}^{\infty} i ! \alpha_{i}(c)^{2} \stackrel{(5.2)}{=} \sum_{i=I}^{\infty} \frac{e^{-c^{2}} \mathrm{He}_{i}^{2}(c)}{2 \pi(i+1) !} .
$$

Since the above upper bound is decreasing in $I$ and in view of the advertised upper bound of Theorem 6 , it is enough to consider the case of $I$ even, i.e. $I=2 N$ for some $N \in \mathbb{N}^{*}$. For such $I$, by Corollary 5 , we have

$$
\begin{aligned}
\sum_{i=2 N}^{\infty} \frac{e^{-c^{2}} \mathrm{He}_{i}^{2}(c)}{2 \pi(i+1) !} & =\frac{1}{2 \pi} \sum_{p=N}^{\infty}\left(\frac{e^{-c^{2}} \mathrm{He}_{2 p}^{2}(c)}{(2 p+1) !}+\frac{e^{-c^{2}} \mathrm{He}_{2 p+1}^{2}(c)}{(2 p+2) !}\right) \\
& \leq \frac{1}{2 \pi} \sum_{p=N}^{\infty}\left(M_{2 p}+M_{2 p+1}\right) \\
& \leq \frac{1}{\pi} \sum_{p=N}^{\infty} M_{2 p}=\frac{1}{\pi} \sum_{p=N}^{\infty} \frac{(2 p) !}{(2 p+1)(p !)^{2} 2^{2 p}}
\end{aligned}
$$

Now using Stirling's formula $p ! \underset{p \rightarrow \infty}{\sim} \sqrt{2 \pi p}\left(\frac{p}{e}\right)^{p}$, we have :

$$
\frac{(2 p) !}{(2 p+1)(p !)^{2} 2^{2 p}} \underset{p \rightarrow \infty}{\sim} \frac{\sqrt{2 \pi 2 p}\left(\frac{2 p}{e}\right)^{2 p}}{(2 p+1) 2 \pi p\left(\frac{p}{e}\right)^{2 p} 2^{2 p}} \underset{p \rightarrow \infty}{\sim} \frac{1}{2 \sqrt{\pi} p^{\frac{3}{2}}}
$$


Standard computations about comparing tails of series and integrals show that, for any $\alpha>1$,

$$
\sum_{k=N}^{\infty} \frac{1}{p^{\alpha}} \underset{N \rightarrow \infty}{\sim} \frac{1}{\alpha-1} \frac{1}{N^{\alpha-1}} .
$$

Applying the above with $\alpha=3 / 2$ directly gives

$$
\frac{1}{\pi} \sum_{p=N}^{\infty} \frac{(2 p) !}{(2 p+1)(p !)^{2} 2^{2 p}} \underset{N \rightarrow \infty}{\sim} \frac{\mathrm{Cst}}{\sqrt{N}}
$$

whence the announced result.

Owing to (2.21), we can write

$$
\mathcal{L}_{I}=\sum_{i=0}^{I} \varepsilon_{K, i} \operatorname{He}_{i}(Z) \quad \text { with } \quad \varepsilon_{K, i}=\sum_{k=1}^{K} l_{k} \alpha_{i}\left(a_{k} \varepsilon_{k}+b_{k}\right) \frac{\rho_{k}^{i}}{\left|\rho_{k}\right|^{i}},
$$

where $\left(\varepsilon_{k}\right)_{k=1 \ldots K}$ are i.i.d. standard normal random variables and independent from $Z$. The striking property is that the coefficients $\left(\varepsilon_{K, i}\right)_{i \in 0, \ldots, I}$ write as a sum of independent random variables. The next result gives a sufficient condition so that one can replace the coefficients by a Gaussian random vector, with explicit characteristics.

Theorem 7. Let $I \in \mathbb{N}$. For every $k \in\{1, \ldots, K\}$, recall the definitions from (2.3) :

$$
a_{k}=\frac{-\sqrt{1-\rho_{k}^{2}}}{\left|\rho_{k}\right|}, b_{k}=\frac{-\Phi^{-1}\left(p_{k}\right)}{\left|\rho_{k}\right|},
$$

and set for every $i, j \in\{0, \ldots, I\}$ :

$$
m_{K, i}:=\sum_{k=1}^{K} l_{k} \frac{\rho_{k}^{i}}{\left|\rho_{k}\right|^{i}} \mu_{i}\left(a_{k}, b_{k}\right), \quad s_{K, i, j}:=\sum_{k=1}^{K} l_{k}^{2} \sigma_{i, j}\left(a_{k}, b_{k}\right),
$$

with $\mu_{i}(a, b):=\mathbb{E}\left[\alpha_{i}(a X+b)\right]$ and $\sigma_{i, j}(a, b):=\mathbb{C o v}\left(\alpha_{i}(a X+b), \alpha_{j}(a X+b)\right)$ for $a \in \mathbb{R}, b \in \mathbb{R}$ and $X \stackrel{d}{=}$ $\mathcal{N}(0,1)$.

Now, assume that the matrix $s_{K}=\left(s_{K, i, j}: 0 \leq i, j \leq I\right)$ is invertible for large $K$ and that

$$
\left\|s_{K}^{-1}\right\| \sup _{1 \leq k \leq K} l_{k}^{2} \underset{K \rightarrow+\infty}{\longrightarrow} 0 \quad \text { with } \quad\left\|s_{K}^{-1}\right\|:=\sup _{x \in \mathbb{R}^{I+1}, x \neq 0} \frac{\left|s_{K}^{-1} x\right|}{|x|} .
$$

Then, the random chaos coefficients vector $\varepsilon_{K}=\left(\varepsilon_{K, i}\right)_{i \in\{0, \ldots, I\}}$ satisfies the following Central Limit Theorem $(C L T)$ :

$$
s_{K}^{-1 / 2}\left(\varepsilon_{K}-m_{K}\right) \underset{K \rightarrow \infty}{\stackrel{d}{\longrightarrow}} \mathcal{N}\left(0, \operatorname{Id}_{I+1}\right),
$$

where $m_{K}=\left(m_{K, i}\right)_{i \in\{0, \ldots, I\}}$.

The assumption (2.26) is rather a theoretical condition which is not easy to simplify, since it links the losses given default $l_{k}$, the correlations $\rho_{k}$ and the unconditional default probabilities $p_{k}$. If we ignore the covariance term $\sigma_{i, j}($.$) and simplify the variance to 1, s_{K}=\left(\sum_{k=1}^{K} l_{k}^{2}\right) \operatorname{Id}_{I+1}$ and the condition (2.26) becomes

$$
\frac{\sup _{1 \leq k \leq K} l_{k}^{2}}{\sum_{k=1}^{K} l_{k}^{2}} \underset{K \rightarrow+\infty}{\longrightarrow} 0
$$


which is, to our opinion, the main take-home message of (2.26): the Gaussian approximation holds if none of the individual square loss is significantly too large compared to the global sum.

From (2.27), as $K$ is assumed to be large, it is natural to approximate the vector $\varepsilon_{K}=\left(\varepsilon_{K, i}\right)_{i \in\{0, \ldots, I\}}$ with a normal vector of mean $m_{K}$ and covariance matrix $s_{K}$. The larger the number of obligors $K$, the better the normal approximation will be. Besides, explicit formulas for $\mu_{i}(),. \sigma_{i, j}($.$) are given in Section 3.1,$ yielding thus explicit expressions for $m_{K}$ and $s_{K}$. As these characteristics can be computed off-line, sampling the meta-model thus reduces to sampling a $I$-dimensional Gaussian vector and the systemic risk factor $Z$, making our approach much faster (see Section 3.2 for further details and remarks on the implementation of our model).

Proof of Theorem \%. We take $K$ large enough, so that $s_{K}$ is invertible and we can define properly $s_{K}^{-1 / 2}$. It is then straightforward to check that the vector $\varepsilon_{K}$ has mean $m_{K}$ and covariance matrix $s_{K}$. Since $s_{K}^{-1 / 2}\left(\varepsilon_{k}-m_{K}\right)$ is a sum of independent vectors, has zero mean and unit covariance matrix, the announced CLT (2.27) is a direct consequence of the CLT for arrays of sums of independent random variables as soon as we check Lindeberg condition (see [BR10, Theorem 18.2] or [JS13, Theorem VII.5.2]): i.e., for any $\varepsilon>0$ and $i \in\{0, \ldots, I\}$, we need :

$$
\sum_{k=1}^{K} l_{k}^{2} \mathbb{E}\left[\left|s_{K}^{-1 / 2} \alpha_{i}\left(a_{k} \varepsilon_{k}+b_{k}\right)-s_{K}^{-1 / 2} \mu\left(a_{k}, b_{k}\right)\right|^{2} \mathbb{1}_{\mathbb{R}_{+}}\left(l_{k}^{2}\left|s_{K}^{-1 / 2} \alpha_{i}\left(a_{k} \varepsilon_{k}+b_{k}\right)-s_{K}^{-1 / 2} \mu\left(a_{k}, b_{k}\right)\right|^{2}-\varepsilon\right)\right] \underset{K \rightarrow \infty}{\longrightarrow} 0 .
$$

On the one hand, observe that

$$
\begin{aligned}
\left\{l_{k}^{2}\left|s_{K}^{-1 / 2} \alpha_{i}\left(a_{k} \varepsilon_{k}+b_{k}\right)-s_{K}^{-1 / 2} \mu\left(a_{k}, b_{k}\right)\right|^{2} \geq \varepsilon\right\} & \subset\left\{l_{k}^{2}\left\|s_{K}^{-1}\right\|\left|\alpha_{i}\left(a_{k} \varepsilon_{k}+b_{k}\right)-\mu\left(a_{k}, b_{k}\right)\right|^{2} \geq \varepsilon\right\} \\
& \subset\left\{\left\|s_{K}^{-1}\right\| \sup _{1 \leq k \leq K} l_{k}^{2} \sup _{c, a, b}\left|\alpha_{i}(c)-\mu(a, b)\right|^{2} \geq \varepsilon\right\}
\end{aligned}
$$

where we have used that $\alpha$ and $\mu$ are bounded (by a constant depending only on the number of coefficients $I+1)$. On the other hand, owing to $(2.26)$, for large $K$ the above event is empty. Therefore, the Lindeberg condition is obviously satisfied and this concludes the proof.

In light of the above Theorem, we define the Gaussian approximation of the I-chaos decomposition by :

$$
\mathcal{L}_{I}^{\mathrm{G}}=\sum_{i=0}^{I} \varepsilon_{K, i}^{\mathrm{G}} \mathrm{He}_{i}(Z) \quad \text { where } \quad \varepsilon_{K}^{\mathrm{G}}=\left(\varepsilon_{K, i}^{\mathrm{G}}\right)_{i=0 \ldots I} \stackrel{d}{=} \mathcal{N}\left(m_{K}, s_{K}\right),
$$

where we recall that both $m_{K}=\left(m_{K, i}\right)_{i=0 \ldots I}$ and $s_{K}=\left(s_{K, i, j}\right)_{i, j=0 \ldots I}$ have been defined in $(2.25)$.

\section{Implementation of the model}

\subsection{Main analytical formulas}

Proposition 8. Let $a \in \mathbb{R}^{*}, b \in \mathbb{R}$ and $X$ a standard normal random variable. For every $i, j \in \mathbb{N}$, define

$$
\begin{aligned}
\mu_{i}(a, b) & :=\mathbb{E}\left[\alpha_{i}(a X+b)\right], \\
\sigma_{i, j}(a, b) & :=\mathbb{C o v}\left(\alpha_{i}(a X+b), \alpha_{j}(a X+b)\right) .
\end{aligned}
$$

Then the following recursive relations hold:

$$
\left\{\begin{array}{l}
\mu_{0}(a, b)=\Phi\left(-\frac{b}{\sqrt{1+a^{2}}}\right), \quad \mu_{1}(a, b)=\frac{e^{-\frac{b^{2}}{2\left(1+a^{2}\right)}}}{\sqrt{2 \pi} \sqrt{1+a^{2}}} \\
\mu_{i+2}(a, b)=\frac{b}{(i+2)\left(1+a^{2}\right)} \mu_{i+1}(a, b)-\frac{i}{(i+2)(i+1)\left(1+a^{2}\right)} \mu_{i}(a, b)
\end{array}\right.
$$




$$
\left\{\begin{array}{l}
\sigma_{0,0}(a, b)=\Phi_{\Sigma}\left((-b,-b)^{\top}\right)-\mu_{0}(a, b)^{2}, \quad \Sigma=\left(\begin{array}{cc}
1+a^{2} & a^{2} \\
a^{2} & 1+a^{2}
\end{array}\right), \\
\sigma_{0,1}(a, b)=\mu_{1}(a, b)\left(\mu_{0}\left(\frac{a}{\sqrt{1+a^{2}}}, \frac{b}{1+a^{2}}\right)-\mu_{0}(a, b)\right), \\
\sigma_{0, i+2}(a, b)=\frac{b}{(i+2)\left(1+a^{2}\right)} \sigma_{0, i+1}(a, b)-\frac{i}{(i+1)(i+2)\left(1+a^{2}\right)} \sigma_{0, i}(a, b)-\frac{a^{2}}{(i+2)\left(1+a^{2}\right)} \mu_{1}(a, b) \mu_{i+1}\left(\frac{a}{\sqrt{1+a^{2}}}, \frac{b}{1+a^{2}}\right), \\
\sigma_{i+1, j+1}(a, b)=\frac{1}{a^{2}(i+1)}\left[-\left(1+a^{2}\right)(j+2) \sigma_{i, j+2}(a, b)+b \sigma_{i, j+1}(a, b)-\frac{j}{j+1} \sigma_{i, j}(a, b)\right]-\mu_{i+1}(a, b) \mu_{j+1}(a, b) .
\end{array}\right.
$$

We exclude the case $a=0$ from the statement since in that case we directly get $\mu_{i}(a, b)=\alpha_{i}(b)$, $\sigma_{i, j}(a, b)=0$. In the non-trivial case $a \neq 0$, the above result gives an effective scheme to compute all the parameters of the meta-model. If the coefficients related to $i, j \in\{0, \ldots, I\}$ are sought, then one shall start with getting $\mu_{i}(a, b)$ from $i=0$ to $I$ using the above initialization and iteration. Then, one shall compute $\sigma_{0, j}(a, b)$ from $j=0$ to $I$. The computation of $\sigma_{i+1, j}(a, b)$ from the $\sigma_{i, .}(a, b)$ is more difficult: since one needs (in particular) $\sigma_{i, j+2}(a, b)$, and by iteration $\sigma_{i-l, j+l+2}(a, b)$ for $l=0$ to $i$. It means that the previous computations for $\mu_{i}(a, b)$ and $\sigma_{0, j}(a, b)$ have to be carried out for $i, j=0, \ldots, 2 I$, in order to be able, at the end, to get $\sigma_{i, j}(a, b)$ for all $i, j=0, \ldots, I$.

The computation by induction of the means $\mu_{i}(a, b)$, the variances $\sigma_{i, i}(a, b)$, the covariances $\sigma_{i, j}(a, b)$ is very simple from the algorithmic point of view. However, for evaluations with $I$ large and for many different $(a, b)$ (the default parameters), we may prefer to have explicit formulas, in order to get possibly more efficient schemes according to the way this is implemented on a computer. The following results are aimed at giving explicit representations of these coefficients as multiple sums, that are straightforward to compute without induction. Note that the expressions below need only to be stated for $i, j \neq 0$. Indeed from Proposition 8 , the case $i=0$ and/or $j=0$ has already be treated as we have derived a closed-form expression for $\mu_{0}(a, b)$ and a recurrence relation for $\sigma_{0, j}(a, b)=\sigma_{j, 0}(a, b)$ for $j \in\{0, \ldots, I\}$.

Lemma 9. For every $i, j \in \mathbb{N}^{*}, \mu_{i}(a, b), \sigma_{i, j}(a, b)$ can be expressed explicitly as :

$$
\begin{aligned}
\mu_{i}(a, b) & =\frac{e^{-\frac{b^{2}}{2\left(1+a^{2}\right)}}}{i \sqrt{2 \pi}} \sum_{k=0}^{\left\lfloor\frac{i-1}{2}\right\rfloor} \frac{a^{2 k} \mathrm{He}_{i-1-2 k}\left(\frac{b}{1+a^{2}}\right)}{2^{k} k !(i-1-2 k) !\left(1+a^{2}\right)^{k+1 / 2}}, \\
\sigma_{i, j}(a, b) & =\frac{e^{-\frac{b^{2}}{1+2 a^{2}}}}{2 \pi i ! j ! \sqrt{1+2 a^{2}}}\left(\sum_{k=0}^{\left\lfloor\frac{i-1}{2}\right\rfloor\left\lfloor\frac{j-1}{2}\right\rfloor} \sum_{p=0}^{i-1} \begin{array}{c}
i \\
2 k
\end{array}\right)\left(\begin{array}{c}
j-1 \\
2 p
\end{array}\right) \frac{a^{2(k+p)}(2(k+p)) ! \mathrm{He}_{i-1-2 k}\left(\frac{b}{1+2 a^{2}}\right) \mathrm{He}_{j-1-2 p}\left(\frac{b}{1+2 a^{2}}\right)}{\left(1+2 a^{2}\right)^{k+p} 2^{k+p}(k+p) !} \\
& +\sum_{k=0}^{\left\lfloor\frac{i-2}{2}\right\rfloor\left\lfloor\frac{j-2}{2}\right\rfloor}\left(\begin{array}{c}
i-1 \\
2 k+1
\end{array}\right)\left(\begin{array}{c}
j-1 \\
2 p+1
\end{array}\right) \frac{a^{2(k+p+1)}(2(k+p+1)) ! \mathrm{He}_{i-2 k-2}\left(\frac{b}{1+2 a^{2}}\right) \mathrm{He}_{j-2 p-2}\left(\frac{b}{1+2 a^{2}}\right)}{\left(1+2 a^{2}\right)^{k+p+1} 2^{k+p+1}(k+p+1) !} \\
& -\mu_{i}(a, b) \mu_{j}(a, b) .
\end{aligned}
$$

\subsection{Numerical tests}

We first focus our attention to the approximation of the indicator funcion $\mathbb{1}_{c \leq Z} \approx \sum_{i=0}^{I} \alpha_{i}(c) \mathrm{He}_{i}(Z)$ and illustrate it with several plots. Keeping in mind that the random variable $Z$ is standard normal, we restrict our attention to $[-3,3]$ for the possible values of $Z$ (more precisely $\mathbb{P}(Z \in[-3,3]) \approx 0.997$ ). Concerning the threshold $c$, note that in our framework it takes values in $\mathbb{R}$. Indeed in light of (2.3),

$$
c=a_{k} \varepsilon_{k}+b_{k} \stackrel{d}{=} \mathcal{N}\left(b_{k}, a_{k}^{2}\right)
$$

The values taken by the normally distributed threshold $c$ thus fully depend on both the values of the default probability $p_{k}$ and the correlation $\rho_{k}$ for the $k$ th obligor. 


\subsubsection{Values taken by the threshold $c$}
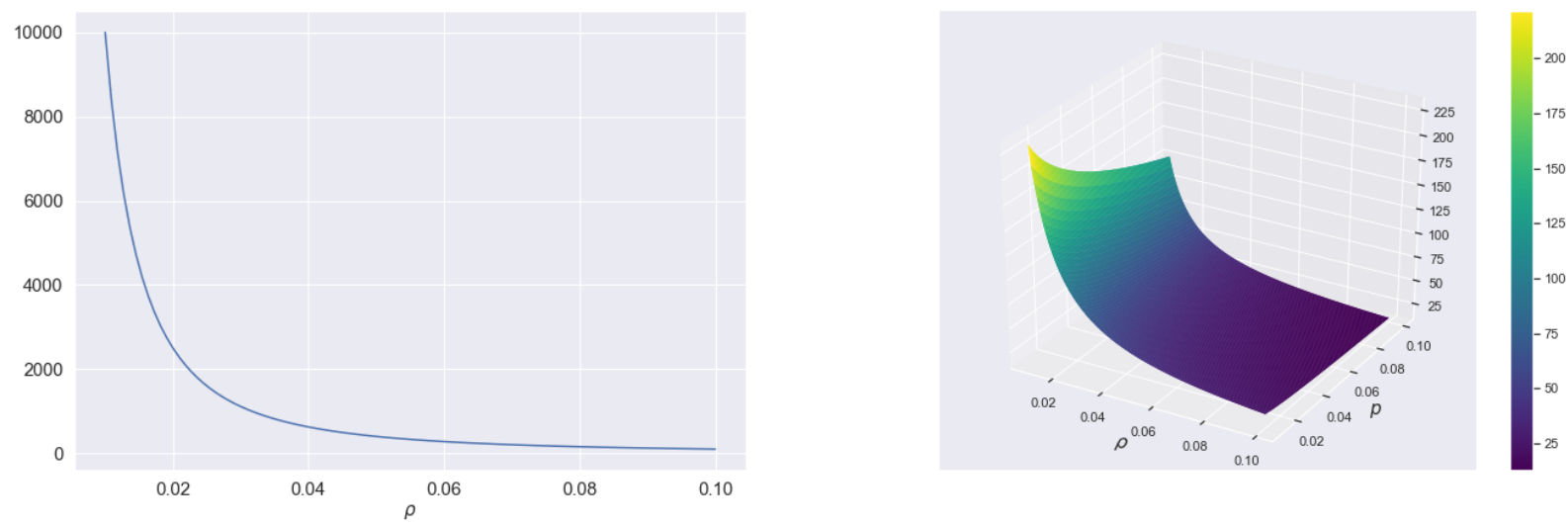

Figure 3: On the left, $\rho \mapsto \frac{1-\rho^{2}}{\rho^{2}}$. On the right, $(\rho, p) \mapsto \frac{-\Phi^{-1}(p)}{\rho}$.

On Figure 3, we plot (omitting the dependence of $k$ ) the mean $(\rho, p) \mapsto \frac{-\Phi^{-1}(p)}{|\rho|}$ and the variance $\rho \mapsto \frac{1-\rho^{2}}{\rho^{2}}$ for positive correlations (similar analysis would be obtained for negative correlation). From both plots, we see that extreme values are obtained with either small correlation and/or small default probability. Economically, this can be interpreted as follows : under such regimes (high values for $c$ ), we see that for the event $\{c \leq Z\}$ to occur, one needs to have an extreme economic scenario (high values for $Z$ ). Indeed as the obligor would (almost) not depend on the econonomy and/or would have a very small default probability, only extreme values of $Z$ (most likely during a crisis) may lead to default.

\subsubsection{Approximation of the indicator $\mathbb{1}_{c \leq Z}$ w.r.t. $Z$}

To visualize the approximation for the indicator function, on Figure 4 we plot for 4 different values of fixed $c \in\{-1,0,1,2\}$, the $I$-chaos decomposition associated where $I \in\{5,20,50,80\}$. We see that as $I$ increases, the quality of the approximation improves together with an increasing number of oscillations and lower amplitudes; the $L_{2}$ convergence does not imply the uniform convergence (Gibbs phenomenon at the discontinuity point, see [San91, Section IV.10]). We notice also that the value $c$ introduces a dissymetry on the quality of the approximation. Indeed if $c<0$ (resp. $c>0$ ), the approximation seems much accurate for $Z>c$ (resp. $Z<c$ ) while for $c=0$ the approximation remains the same.

There is another phenomena worth mentioning when one approximates the indicator function (bounded function) using the Wiener chaos decomposition. As we are approximating the indicator function with a sum of Hermite polynomials (non-bounded functions), one has that for large values of $Z$, the quality of the approximation deteriorates completely getting extreme amplitudes (see Figure 5). However as said before, $Z$ is standard normal and such values for $Z$ are very unlikely. Let us also notice that the rough estimate obtained in Theorem 6 allows us to cover a large range of values for the threshold $c$ though the estimate could presumably be refined for certain range of values.

Finally in order to illustrate the $L_{2}$ error in Theorem 6 , we provide the log-log plot of the mean square error $\mathrm{MSE}=\mathbb{E}\left[\left|\mathbb{1}_{c \leq Z}-\sum_{i=0}^{I} \alpha_{i}(c) \mathrm{He}_{i}(Z)\right|^{2}\right]$ w.r.t. $I$ for 4 different values of fixed $c \in\{-1,0,1,2\}$. More precisely, we fix $I=50$, generate $N=5 \times 10^{5}$ i.i.d. samples $\left(Z_{n}\right)_{1 \leq n \leq N} \stackrel{d}{=} \mathcal{N}(0,1)$ and estimate the MSE by $\mathrm{MC}$ simulation. As expected, we retrieve the error like Cst $/ \sqrt{I}$ since $\log (\mathrm{MSE})$ is a linear function w.r.t. $\log (I)$ with a slope of $-1 / 2$ (see Figure 6). Actually note that in light of Theorem 6 , we have a closed 

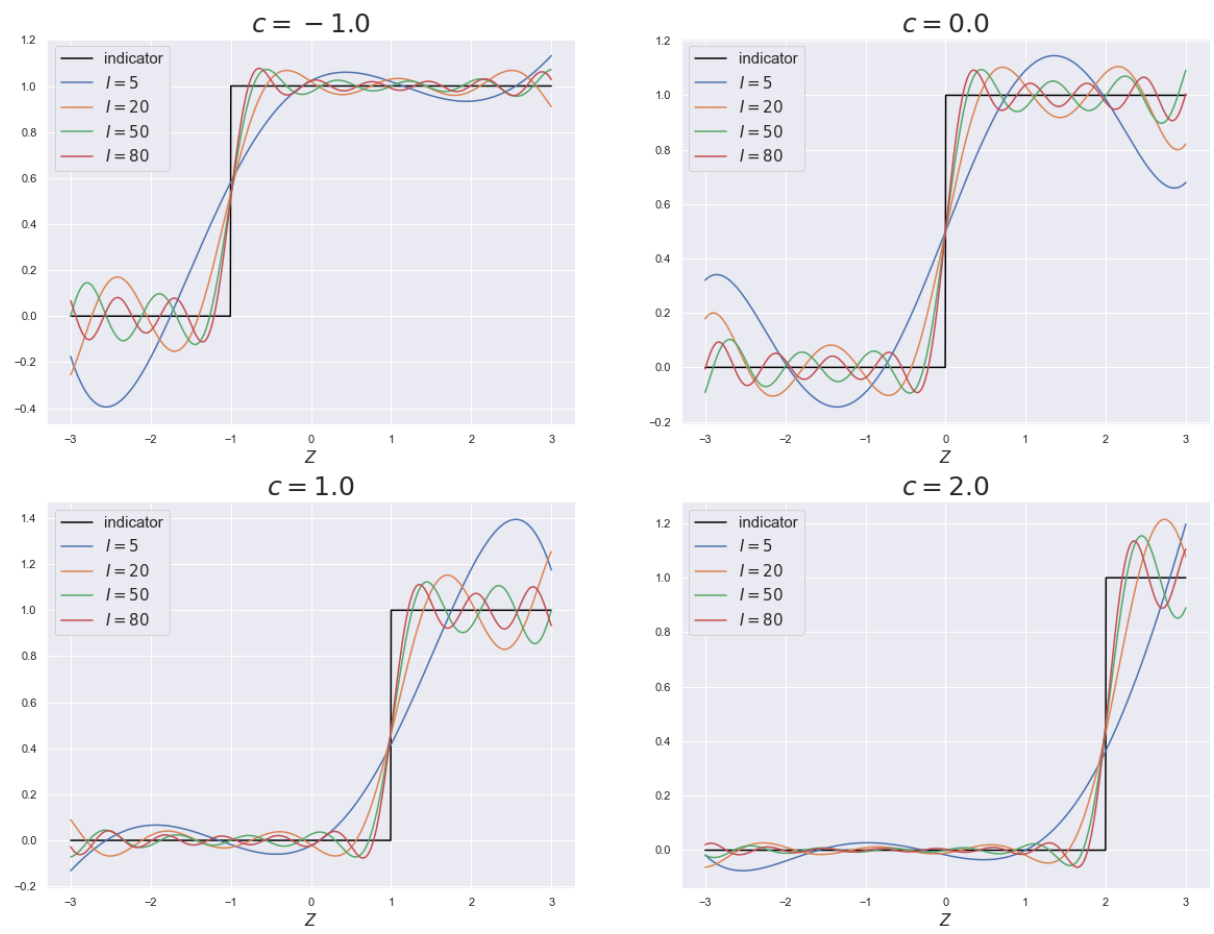

Figure 4: $I$-chaos decompositons for $\mathbb{1}_{c \leq Z}$ with $I \in\{5,20,50,80\}$ and $c \in\{-1,0,1,2\}$

formula for the MSE i.e MSE $=\sum_{i=I+1}^{\infty} i ! \alpha_{i}(c)^{2}$. However in order to avoid numerical instability by trying to compute Hermite polynomials of very high degree (over $i=200$, errors are returned), we prefer to use a MC simulation to evaluate numerically the MSE.

\subsubsection{The portfolio loss and its chaos decomposition}

Let us recall that the $I$-chaos decomposition (2.21) and its Gaussian-based approximation (2.29) for the portfolio loss are given by

$$
\mathcal{L}_{I}=\sum_{i=0}^{I} \varepsilon_{K, i} \mathrm{He}_{i}(Z)=\sum_{i=0}^{I}\left\{\sum_{k=1}^{K} l_{k} \alpha_{i}\left(a_{k} \varepsilon_{k}+b_{k}\right) \frac{\rho_{k}^{i}}{\left|\rho_{k}^{i}\right|}\right\} \mathrm{He}_{i}(Z), \quad \mathcal{L}_{I}^{\mathrm{G}}=\sum_{i=0}^{I} \varepsilon_{K, i}^{\mathrm{G}} \mathrm{He}_{i}(Z),
$$

where $a_{k} \varepsilon_{k}+b_{k} \stackrel{d}{=} \mathcal{N}\left(b_{k}, a_{k}^{2}\right), a_{k}=-\frac{\sqrt{1-\rho_{k}^{2}}}{\left|\rho_{k}\right|}, b_{k}=-\frac{\Phi^{-1}\left(p_{k}\right)}{\left|\rho_{k}\right|}$ and $\varepsilon_{K}^{\mathrm{G}} \stackrel{d}{=} \mathcal{N}\left(m_{K}, s_{K}\right)$.

To fix ideas, we give below the algorithm to generate $N$ samples from the Gaussian-based approximation $\mathcal{L}_{I}^{\mathrm{G}}$. As stated before, the only parameters (which can be computed off-line) needed, are the mean vector $m_{K}$ and covariance matrix $s_{K}$ that can be either computed using the recurrence relations in Proposition 8 or by explicit expressions stated in Lemma 9. The costly step (with respect to $K$ ) is made off-line when computing $m_{K}$ and $s_{K}$, this is a very significant improvement compared to the crude algorithm where new sums over $k$ have to be made at each sample of $\mathcal{L}$. More precisely, the Gaussian-based approximation allows us to bypass the computation of the sum over all obligors needed in a naive approach for the sampling of $\mathcal{L}$ (see Algorithms 1, 2 and Remark 10). Note that we actually take advantage of the fact that $K$ is very large since the larger the number of obligors, the better the Gaussian-based approximation will be. 


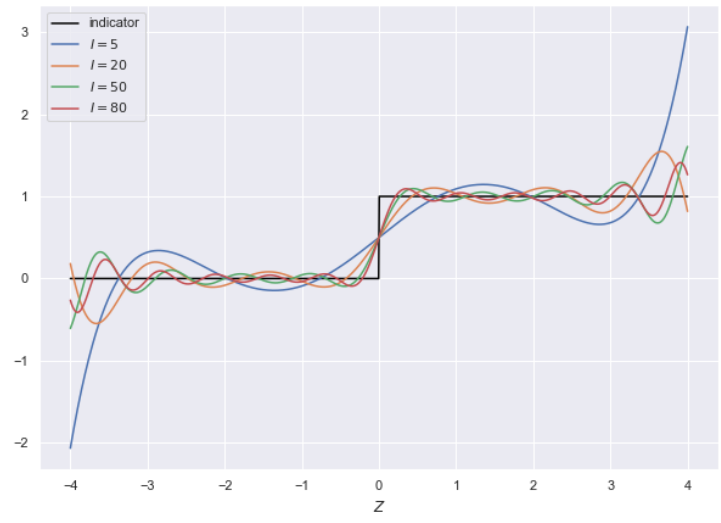

Figure 5: $I$-chaos decompositons for $\mathbb{1}_{0 \leq Z}$ with $I \in\{5,20,50,80\}$ to illustrate extreme amplitudes in the tails
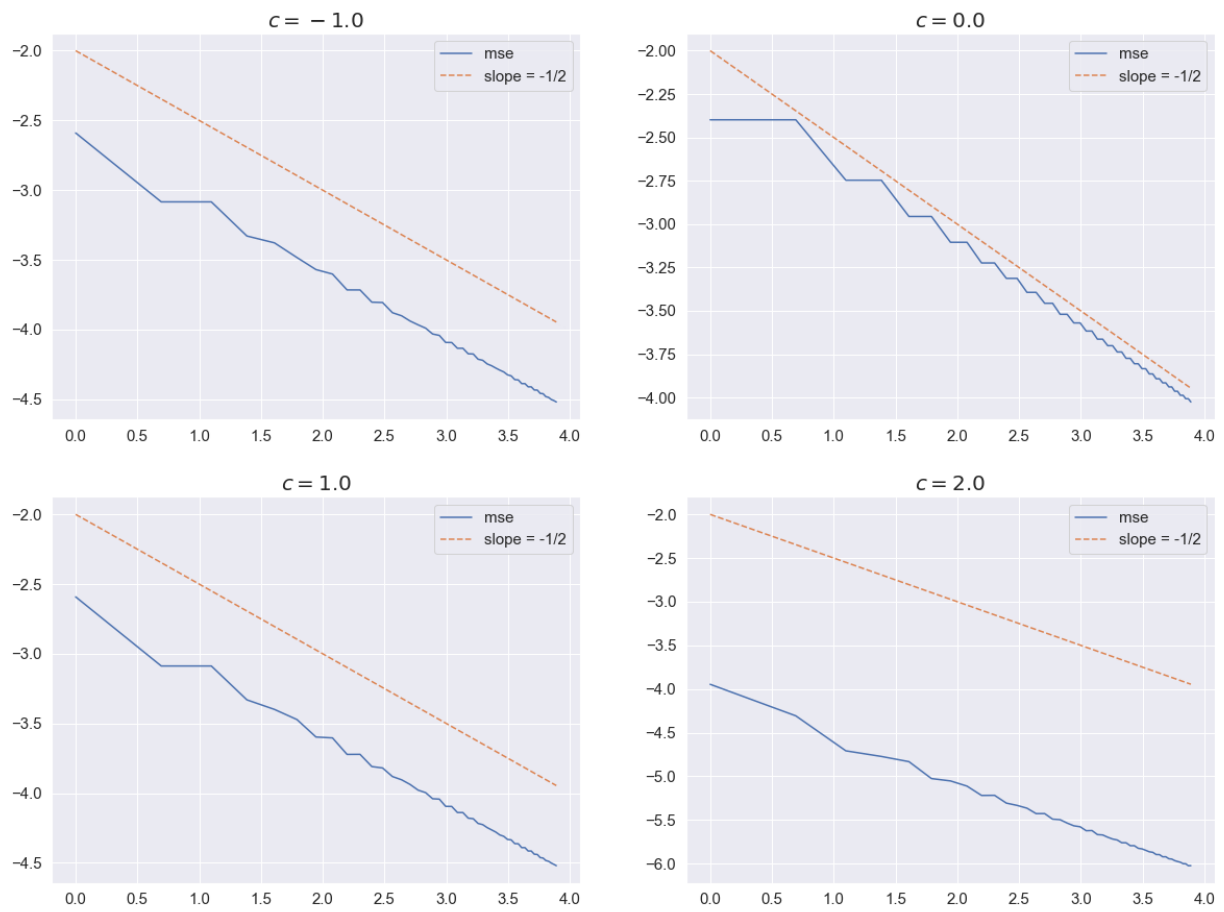

Figure 6: $\log \left(\mathbb{E}\left[\left|\mathbb{1}_{c \leq Z}-\sum_{i=0}^{I} \alpha_{i}(c) \mathrm{He}_{i}(Z)\right|^{2}\right]\right)$ w.r.t. $\log (I)$ for $c \in\{-1,0,1,2\}$ 

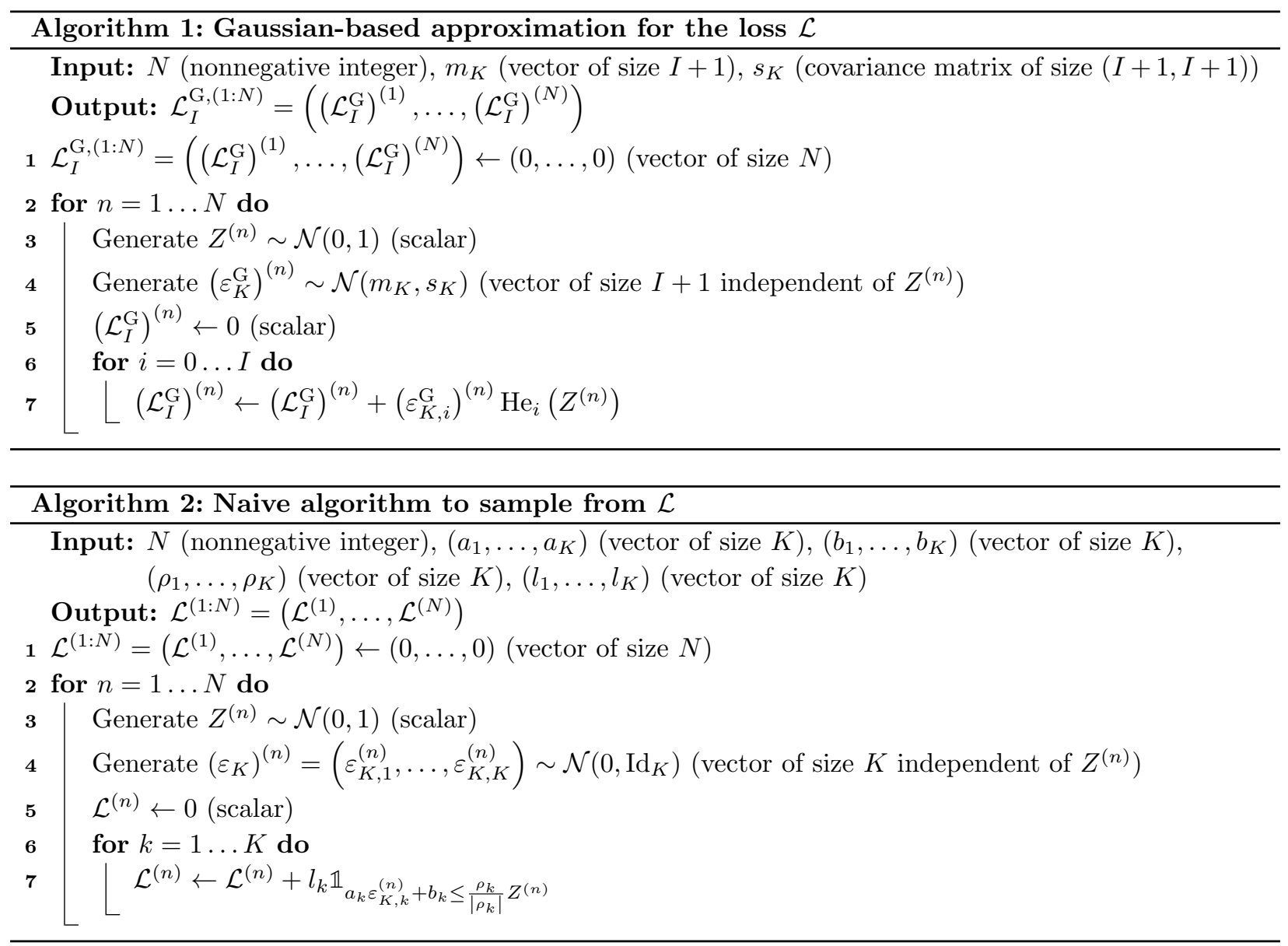

Remark 10. To assess the efficiency of our method, we provide both complexity of the two algorithms, in terms of elementary operations. We assume that any Hermite polynomial of degree $i$ can be computed at one unit cost $\mathcal{O}(1)$ : this is a reasonable assumption since one usually needs to compute these polynomials at once from degree 0 to $I$, which can be done by the recursive relation (2.5). For giving the complexity of Algorithm 1, we separate the offline/online parts for the approximation.

Offline computational cost : From Proposition 8, we see that $\mu_{0}, \mu_{1}$ are explicit (unit cost) and each $\left(\mu_{i}\right)_{i \geq 2}$ requires one unit cost $\mathcal{O}(1)$ owing to the recursive relation (3.1). Hence from the Definition 2.25, computing $m_{K}$ requires $\mathcal{O}(K I)$ operations. A similar analysis gives that the computational cost for $s_{K}$ using (3.2) is $\mathcal{O}\left(K I^{2}\right)$ and $\mathcal{O}\left(I^{3}\right)$ for having a Cholesky decomposition of $s_{K}$. Overall the offline cost is $\mathcal{O}(K I)+$ $\mathcal{O}\left(K I^{2}\right)+\mathcal{O}\left(I^{3}\right)=\mathcal{O}\left(K I^{2}\right)$, using that usually $K \gg I$.

Online computationcal cost : Once a Cholesky decomposition of $s_{K}$ is obtained, sampling $\left(\varepsilon_{K, i}^{\mathrm{G}}\right)_{i=0 \ldots I}$ can be done at cost $\mathcal{O}\left(I^{2}\right)$, which implies that our Gaussian-based algorithm for $N$ samples only requires $\mathcal{O}\left(N I^{2}\right)$ operations.

The total cost of Algorithm 1 is then $\mathcal{O}\left(K I^{2}\right)+\mathcal{O}\left(N I^{2}\right)$. On the contrary, the naive Algorithm 2 would require $\mathcal{O}(N K)$ operations. Remembering that $K \geq 10^{5}$ and $I \leq 50$ (in most cases it is already enough), the computational cost of Algorithm 1 is much smaller.

We now want to test such a decomposition and see how well it approximates the true portfolio loss $\mathcal{L}$. More precisely, we consider different portfolio scenarios $\{A, B\}$ as choices need to be made for the $k$ th obligor correlation $\rho_{k}$, default probability $p_{k}$ and the loss $l_{k}$ together with the values taken by the economy $Z$. We fix the (large) number of obligors at $K=5 \times 10^{5}$. For each scenario, we provide :

- The histogram of $N=10^{4}$ samples $\varepsilon_{K, i}$ together with the normal probability density centered at $m_{K, i}$ and with variance $s_{K, i, i}$ for different $i \in\{1,3,6,8\}$. Indeed for a fixed $i \in\{0, \ldots, I\}$, we know from 
Theorem 7 that $\mathbb{E}\left[\varepsilon_{K, i}\right]=m_{K, i}, \operatorname{Var}\left(\varepsilon_{K, i}\right)=s_{K, i, i}$. We thus want to test how well the $\varepsilon_{K, i}$ marginals are close to a normal $\mathcal{N}\left(m_{K, i}, s_{K, i, i}\right)$.

- The kernel density estimation (k.d.e.) of $\mathcal{L}_{s} I$ conditionally on $Z$ from $N=10^{4}$ samples $\mathcal{L}_{I} \mid Z$ and the 'true' conditional loss $\mathcal{L} \mid Z$ for different economic scenarios $Z \in\{-1,0,1,2\}$ and truncation parameters $I \in\{1,3,6,9\}$.

- The k.d.e. of $\mathcal{L}$ and $\mathcal{L}_{I}^{\mathrm{G}}$ for $I \in\{1,3,6,9\}$ with $N=10^{5}$ samples.

- In order to assess if $\mathcal{L}_{I}^{\mathrm{G}}$ succeeds in approximating $\mathcal{L}$ in the tails, we provide the quantile-quantile plot of $\mathcal{L}_{I}^{\mathrm{G}}$ w.r.t. $\mathcal{L}$ for $I \in\{1,3,6,9,12,15\}$ with $N=10^{5}$ samples.

Portfolio A : We consider both homogeneous correlation and default probability i.e $\forall k \in\{1, \ldots K\}$, $p_{k}=0.01, \rho_{k}=0.1$ and take the $k$ th loss of the form $l_{k}=1 / \sqrt{k}$. Note that in that case, we have that $a_{k}=\frac{-\sqrt{1-\rho^{2}}}{|\rho|}=a, b_{k}=\frac{-\Phi^{-1}(p)}{|\rho|}=b$ and for every $i, j \in\{0, \ldots, I\}, m_{K, i}=\mu_{i}(a, b) \frac{\rho^{i}}{|\rho|^{i}} \sum_{k=1}^{K} l_{k}, \quad s_{K, i, j}=$ $\sigma_{i, j}(a, b) \sum_{k=1}^{K} l_{k}^{2}$. The simplified Lindeberg condition (2.28) easily holds as $\sup _{1 \leq k \leq K} l_{k}^{2}=1$ and $\sum_{k=1}^{K} \frac{1}{k} \underset{K \rightarrow+\infty}{\longrightarrow}+\infty$.
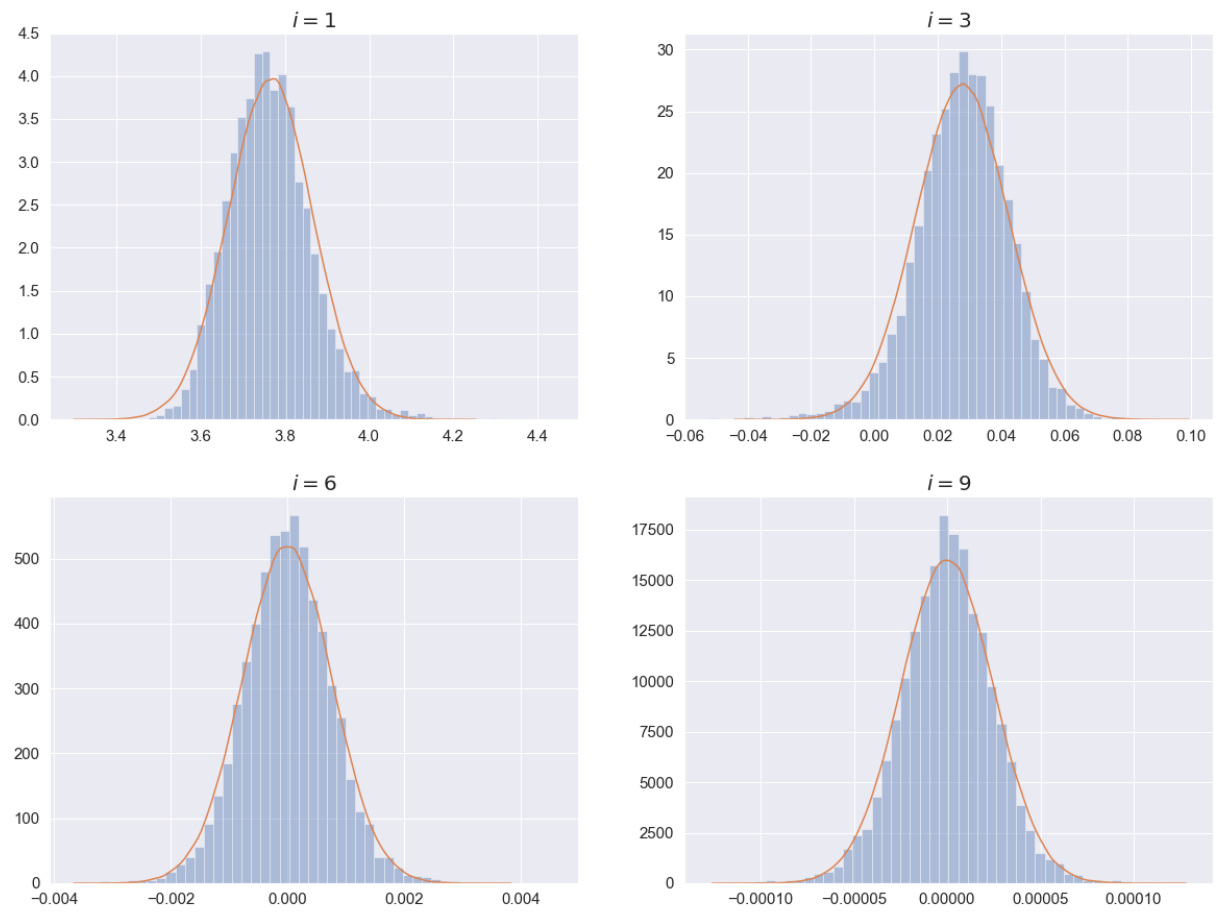

Figure 7: Histograms of $\varepsilon_{K, i}$ with the p.d.f of $\mathcal{N}\left(\mathbb{E}\left[\varepsilon_{K, i}\right], \operatorname{Var}\left(\varepsilon_{K, i}\right)\right)$ for $i \in\{1,3,6,9\}$ for the Portfolio A 

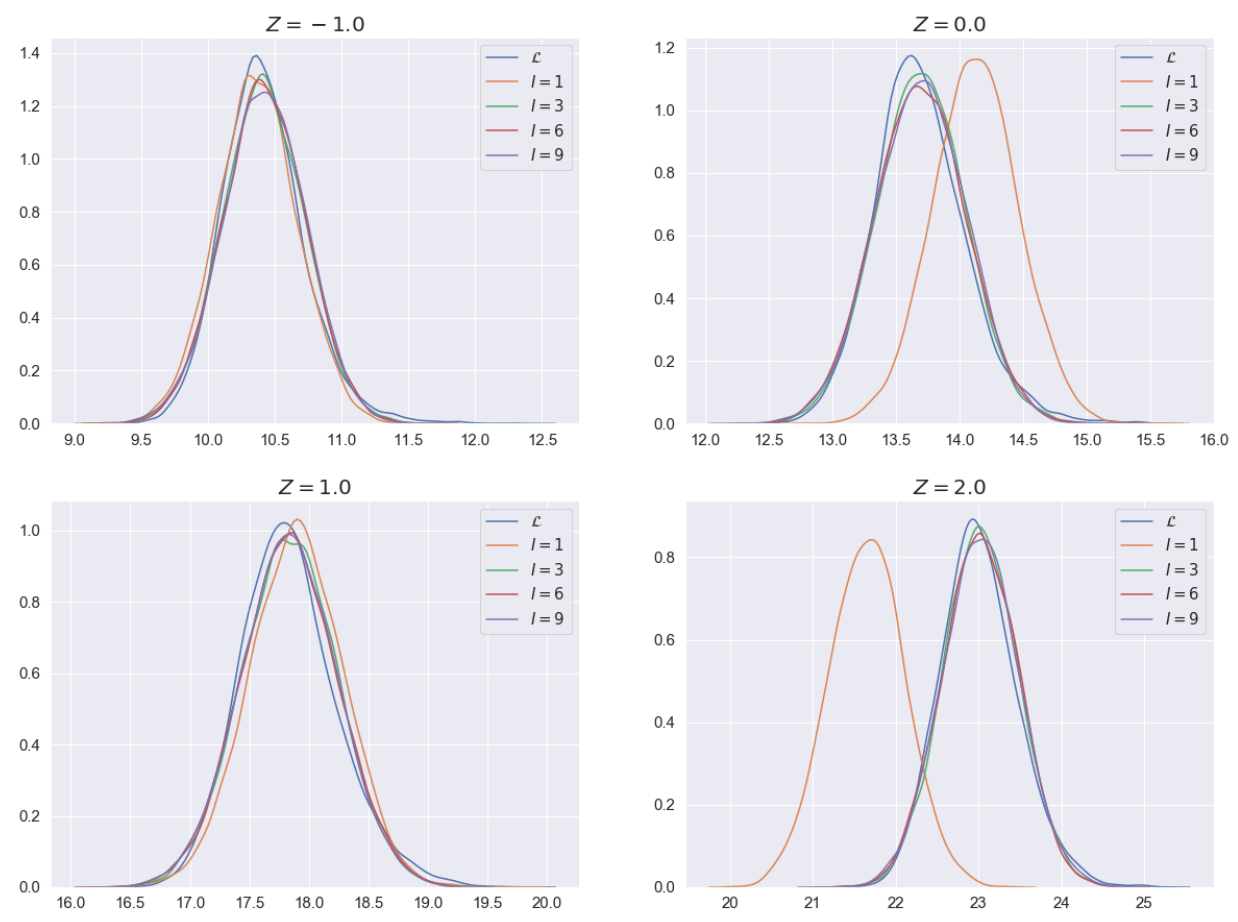

Figure 8: K.d.e of $\mathcal{L}_{I}^{\mathrm{G}}$ for $I \in\{1,3,6,9\}$ and $\mathcal{L}$ conditionally on $Z \in\{-1,0,1,2\}$ for the Portfolio A

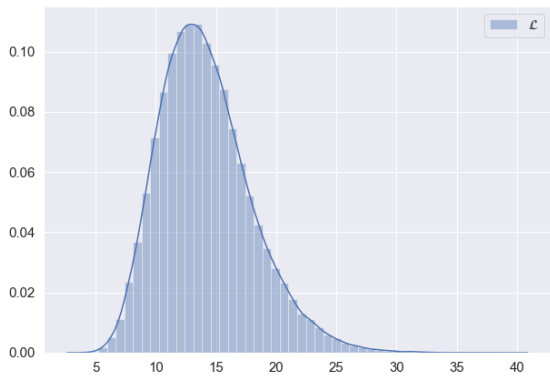

(a) Distribution of $\mathcal{L}$

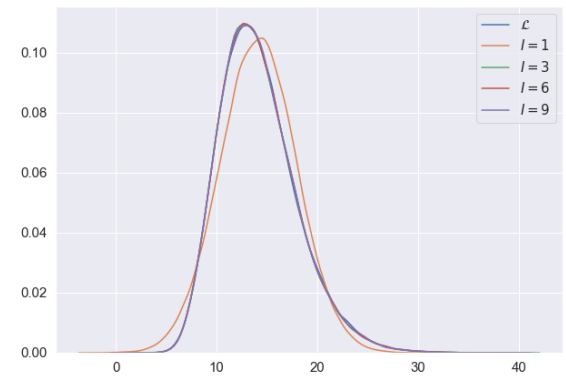

(b) K.d.e of $\mathcal{L}_{I}^{\mathrm{G}}$ for $I \in\{1,3,6,9\}$ and $\mathcal{L}$

Figure 9: Loss of Portfolio A 


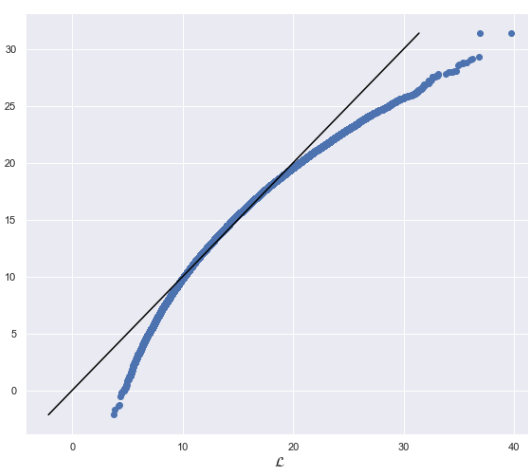

(a) $I=1$

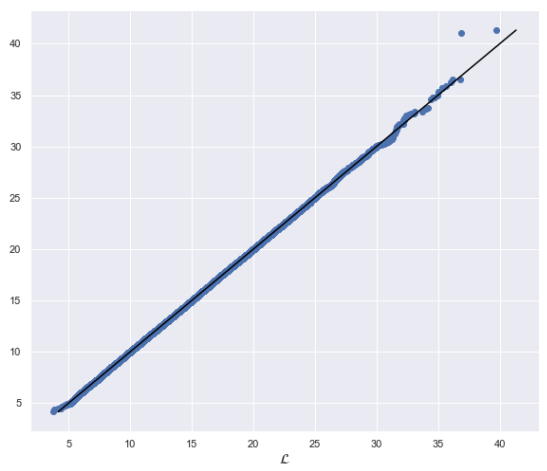

(c) $I=6$

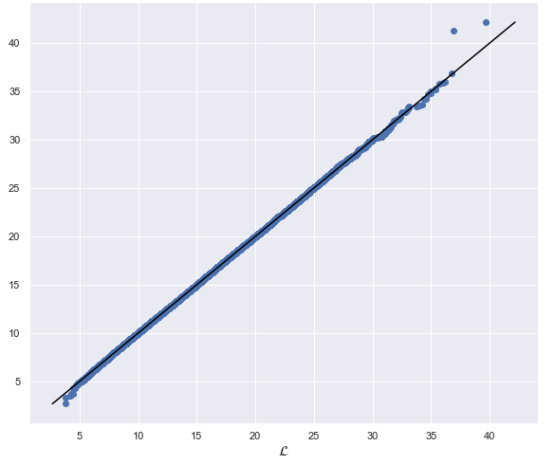

(b) $I=3$

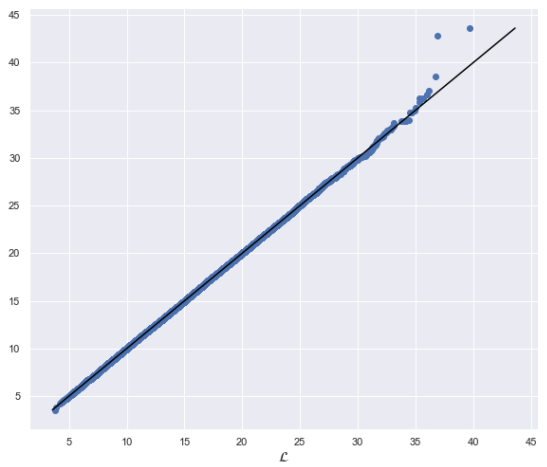

(d) $I=9$

Figure 10: Q-Q plots of $\mathcal{L}_{I}^{\mathrm{G}}$ w.r.t. $\mathcal{L}$ for the Portfolio A

In Figure 7, all marginals $\varepsilon_{K, i}$ for $i=1,3,6,9$ seem to be very close to a normal distribution centered at $m_{K, i}$ and with variance $s_{K, i}$ indicating that a Gaussian approximation seems legit. In both Figures 8 and 9 , we see that the Gaussian-based approximation $\mathcal{L}_{I}^{\mathrm{G}}$ quickly converges to the 'true' loss $\mathcal{L}$ for small $I$ as both densities are indistinguishable from $I=3$. As for the previous graphs, the Q-Q plot in Figure 10 shows a poor approximation for $I=1$. From $I=6$, the two distributions have almost the same estimated quantiles with an error of approximately $(2 / N) \times 100=0.002 \%$ ( 2 quantiles differ totally when $\mathcal{L} \geq 36$ ). Notice also that increasing the truncating parameter from $I=6$ to $I=9$ does not seem to yield any improvement which probably indicates that the error (for those $I$ ) comes from the Gaussian approximation of the $\varepsilon_{K, i}$.

Portfolio B $:$ We consider the same portfolio as in [GL05] i.e $\forall k \in\{1, \ldots K\}, p_{k}=0.01\left(1+\sin \left(\frac{16 \pi k}{K}\right)\right)+$ $0.001, \rho_{k} \stackrel{d}{=} \mathcal{U}([0,1 / \sqrt{10}])+0.001$ (we add 0.001 so that $p_{k} \neq 0$ and $\rho_{k} \neq 0$ for every obligor $k$ ) and $l_{k}=$ $\left(\left\lceil\frac{5 k}{K}\right]\right)^{2}$. Note in that case that the marginal default probabilities vary between $0.001 \%$ and $2.001 \%$ with a mean $1.0005 \%$ and the loss $l_{k}$ are 1,4,9,16,25 with $K / 5$ at each level. Notice also that the simplified Lindeberg condition (2.28) holds since $\sup _{1 \leq k \leq K} l_{k}^{2}=25$ and $\sum_{k=1}^{K}\left(\left\lceil\frac{5 k}{K}\right\rceil\right)^{2} \geq \frac{25}{K^{2}} \sum_{k=1}^{K} k^{2}=\frac{25}{K^{2}} \frac{K(K+1)(2 K+1)}{6} \sim$ $\frac{25}{3} K^{2} \underset{K \rightarrow+\infty}{\longrightarrow}+\infty$. We recall that $\bar{K}=5 \times 10^{5}$. 
$I_{k}$

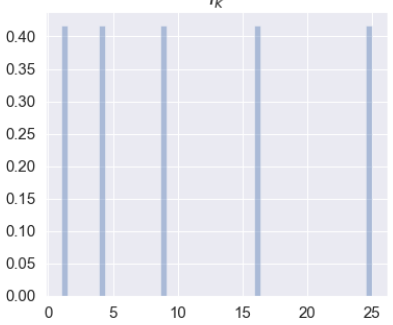

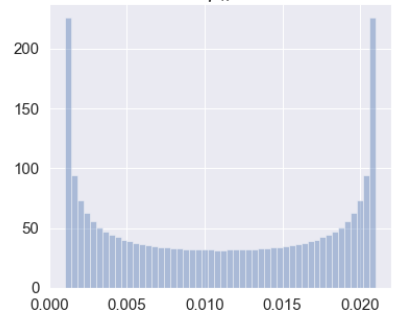

$\rho_{k}$

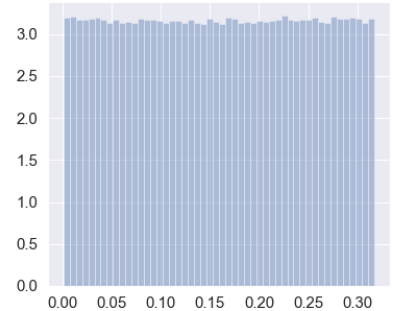

Figure 11: Histograms of $l_{k}, p_{k}, \rho_{k}$ for the Portfolio B
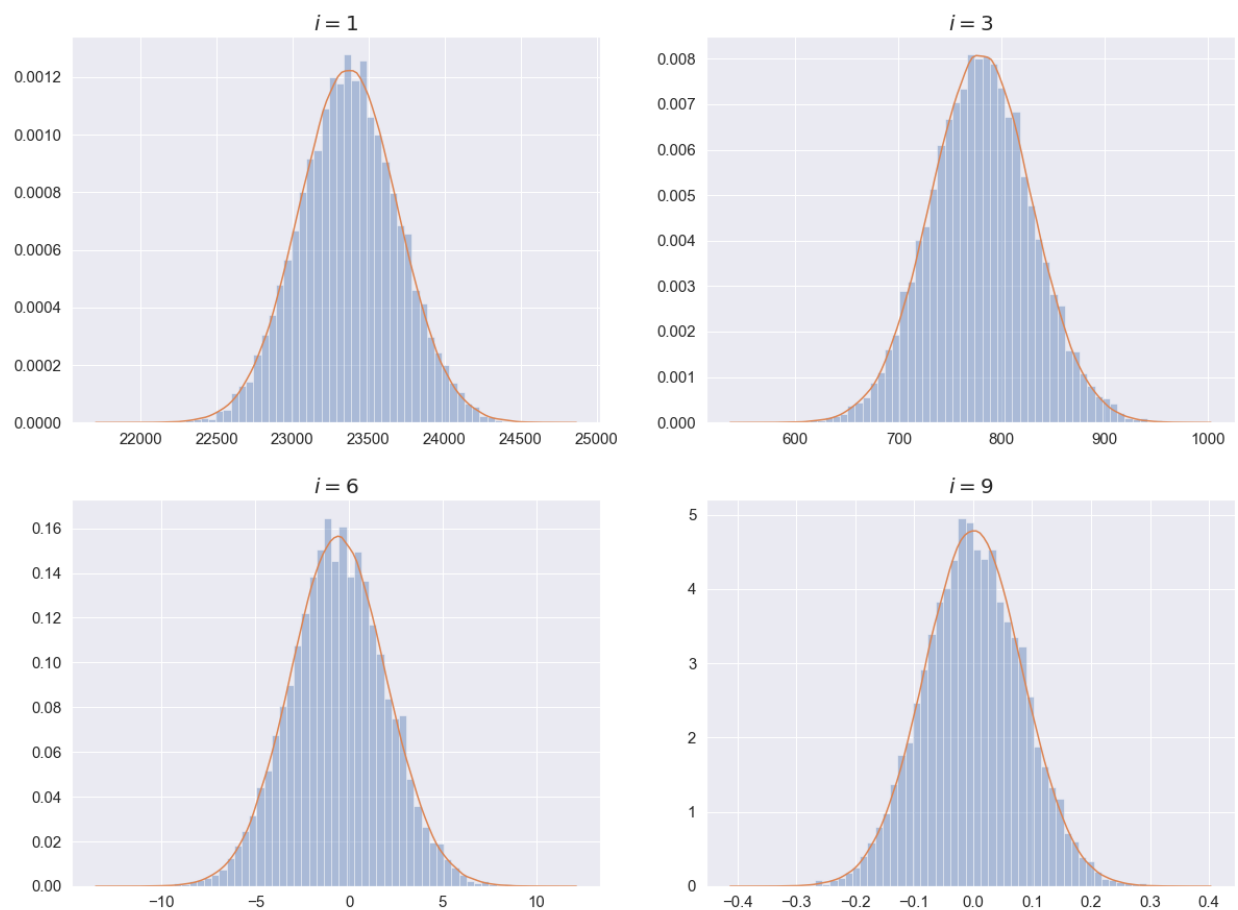

Figure 12: Histograms of $\varepsilon_{K, i}$ with the p.d.f of $\mathcal{N}\left(\mathbb{E}\left[\varepsilon_{K, i}\right]\right.$, $\left.\operatorname{Var}\left(\varepsilon_{K, i}\right)\right)$ for $i \in\{1,3,6,9\}$ for the Portfolio B 

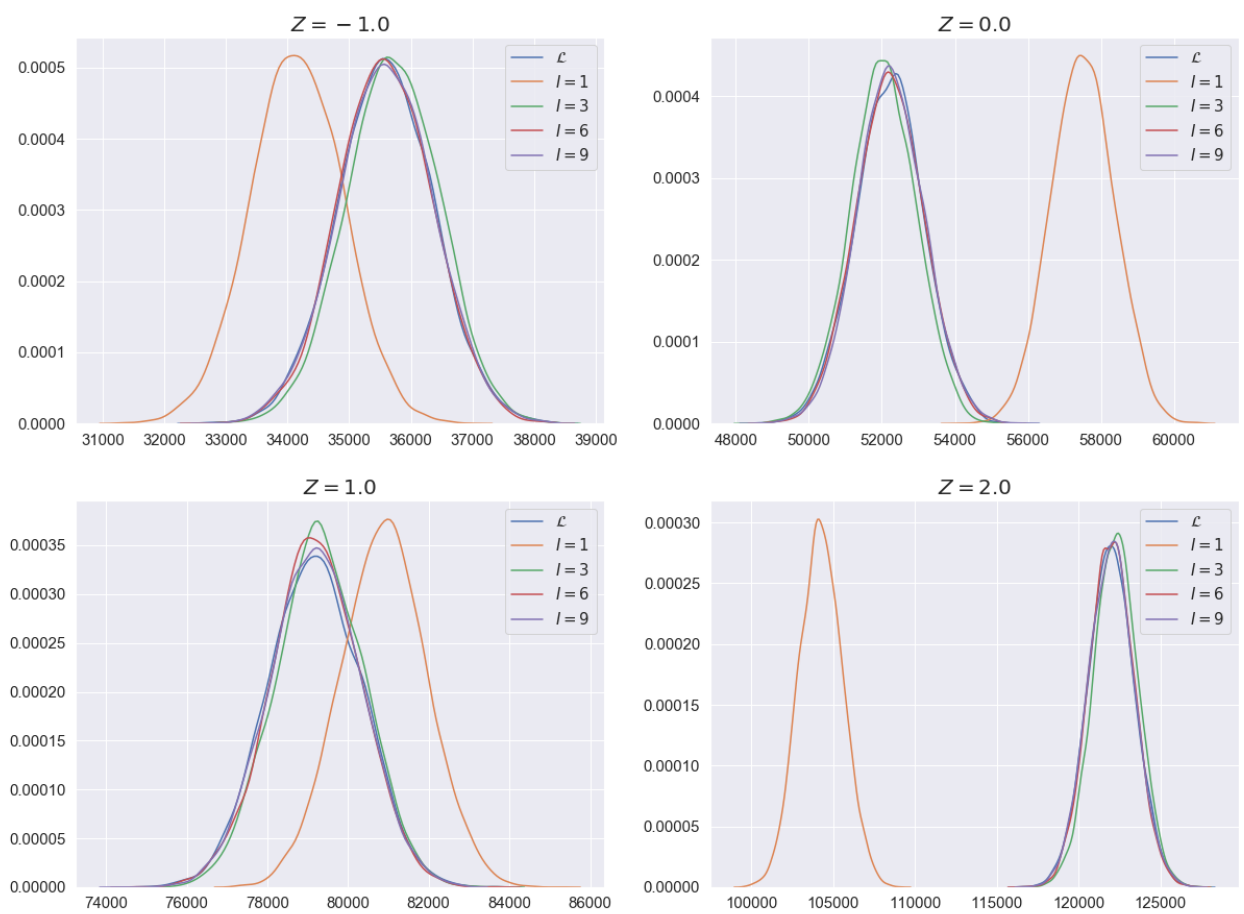

Figure 13: K.d.e of $\mathcal{L}_{I}^{\mathrm{G}}$ for $I \in\{1,3,6,9\}$ and $\mathcal{L}$ conditionally on $Z \in\{-1,0,1,2\}$ for the Portfolio B

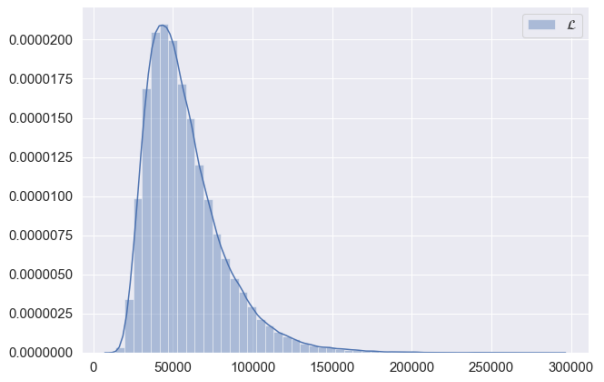

(a) Distribution of $\mathcal{L}$

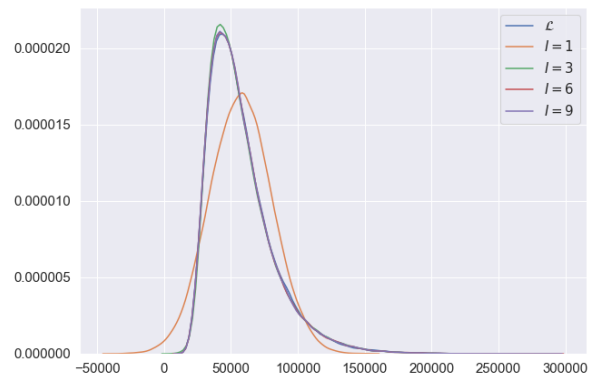

(b) K.d.e of $\mathcal{L}_{I}^{\mathrm{G}}$ for $I \in\{1,3,6,9\}$ and $\mathcal{L}$

Figure 14: Loss of Portfolio B 


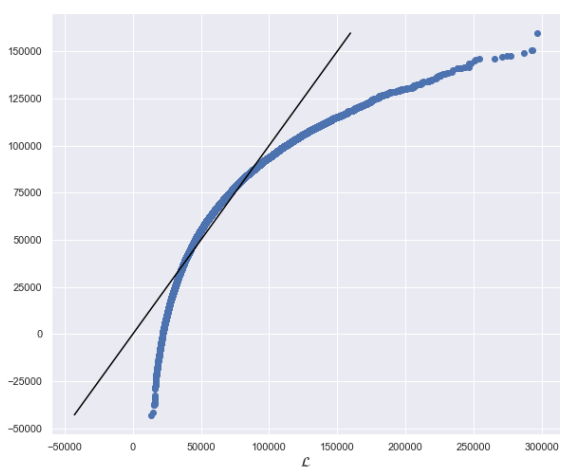

(a) $I=1$

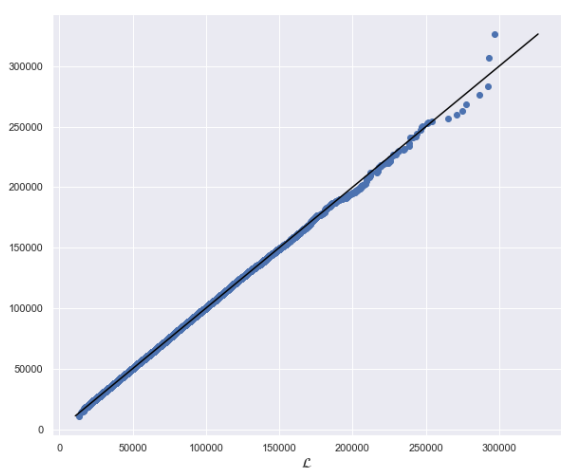

(c) $I=6$

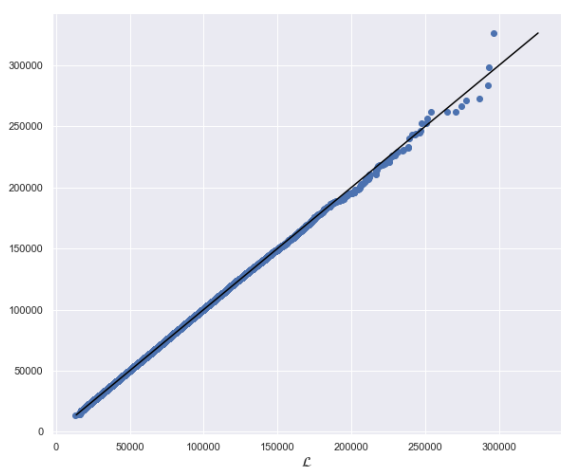

(e) $I=12$

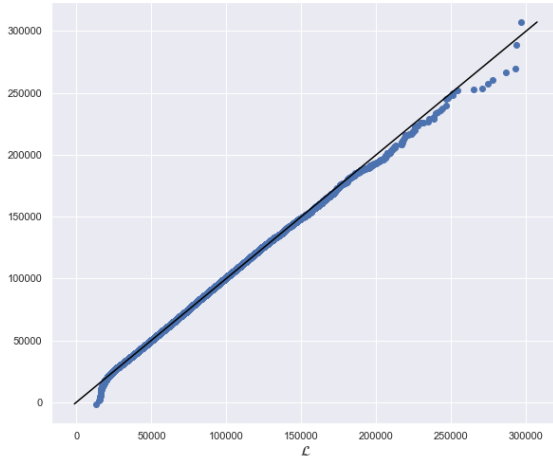

(b) $I=3$

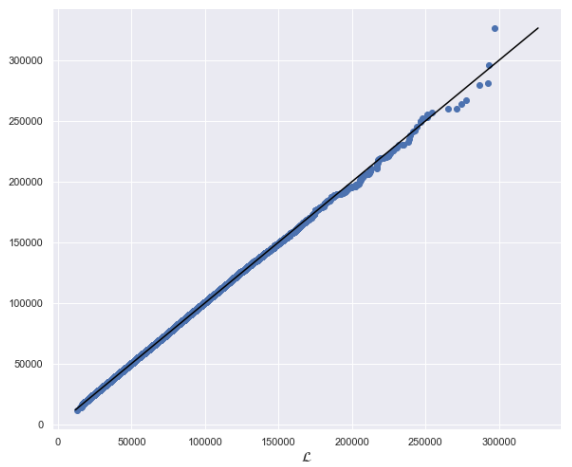

(d) $I=9$

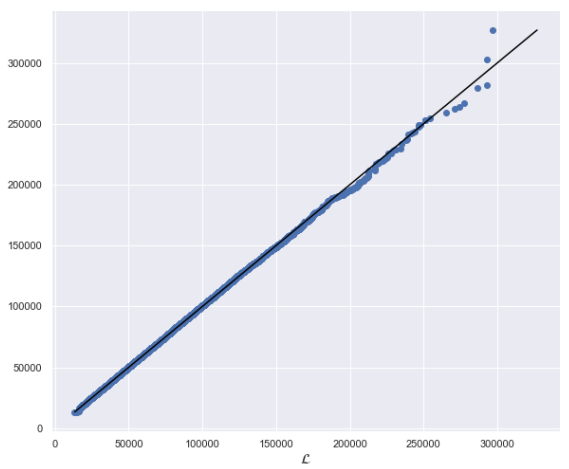

(f) $I=15$

Figure 15: Q-Q plots of $\mathcal{L}_{I}^{\mathrm{G}}$ w.r.t. $\mathcal{L}$ for the Portfolio B

In the simplified portfolio A, our approximation seems to give excellent results. We now want to challenge our method by conducting the same analysis with a less homogenous (and thus more realistic) portofolio. Figures 12, 13 and 14 essentially give the same results as for the Portfolio A where we see that a small $I$ seems to already give satisfying results. For the Q-Q plot of Figure 15 however, we notice that for $I=1,3$ our method is unable to approach accurately the true loss. From $I=6$, our approximation provides quite satisfactory results with an error of approximately $(10 / N) \times 100=0.01 \%$ (10 quantiles differ totally when $\mathcal{L} \geq 250000$ ). Hence, our approach can be used for the computation of risk measures (such as VaR) up to 99.99\% which is probably more than enough for the current regulation. Again notice that increasing the truncating parameter from $I=6$ does not seem to give a better approximation (probably due to the lack of 
perfect accuracy of the Gaussian approximation).

\subsection{Extension to the $d$-factor credit model}

In this section, we extend our results obtained so far to a $d$-factor Gaussian copula model. For the sake of clarity, we introduce the following standard multi-index notation; for multi-indices $\mathbf{i}=\left(i_{1}, \ldots, i_{d}\right), \mathbf{j}=$ $\left(b_{1}, \ldots, b_{d}\right) \in \mathbb{N}_{0}^{d}$ and vector $\mathbf{x}=\left(x_{1}, \ldots, x_{d}\right) \in \mathbb{R}^{d}$, we write :

- $\mathbf{i} \pm \mathbf{j}=\left(i_{1} \pm j_{1}, \ldots, i_{d} \pm j_{d}\right)$ (componentwise sum and difference);

- $|\mathbf{i}|=i_{1}+\cdots+i_{d}$ (sum of components);

- $\mathbf{i} !=i_{1} ! \ldots i_{d}$ ! (factorial)

- $\left(\begin{array}{c}|\mathbf{i}| \\ \mathbf{i}\end{array}\right)=\frac{|\mathbf{i}|}{\mathbf{i} !}=\frac{|\mathbf{i}|}{i_{1} ! \ldots i_{d} !}$ (multinomial coefficient);

- $\mathbf{x}^{\mathbf{i}}=x_{1}^{i_{1}} \ldots x_{d}^{i_{d}}$ (power);

- $\|\mathbf{x}\|=\sqrt{x_{1}^{2}+\cdots+x_{d}^{2}}$ (Euclidean norm).

So far our analysis was built on a one-factor $(d=1)$ model. The extension to a $d$-factor credit model goes as follows (see [GL05]). The default event indicator remains $Y_{k}=\mathbb{1}_{\left\{X_{k} \geq c_{k}\right\}}$ (as for (2.2)), with a stochastic factor $X_{k}$ taking now the form

$$
X_{k}=\boldsymbol{\rho}_{k} \cdot \boldsymbol{Z}+\beta_{k} \varepsilon_{k}=\sum_{j=1}^{d} \rho_{k j} Z_{j}+\beta_{k} \varepsilon_{k}
$$

where the $d$ systemic factors (each representing an industry sector or a geographic zone) $\boldsymbol{Z}=\left(Z_{1}, \ldots, Z_{d}\right)$ are independent standard normal random variable and $\varepsilon_{k}$ is an idiosyncratic risk factor. The sequence $\left(\varepsilon_{k}\right)_{k=1, \ldots, K}$ is made of independent standard normal random variables, and independent from the $d$ systemic factors.

Without loss of generality, we can assume that the coefficients are adjusted so that the stochastic factor $X_{k}$ is standard normal. The factor loadings vector (correlations to the systemic factors) for the $k$ th obligor are denoted by $\boldsymbol{\rho}_{k}=\left(\rho_{k 1}, \ldots, \rho_{k d}\right)$. Since $X_{k}$ is standard normal, we can set $\beta_{k}=\sqrt{1-\left\|\boldsymbol{\rho}_{k}\right\|^{2}}$. The constraint $p_{k}=\mathbb{P}\left(Y_{k}=1\right)$ gives again

$$
c_{k}=-\Phi^{-1}\left(p_{k}\right)
$$

We now give the extension to both Theorem 6 and 7 in the $d$-factor Gaussian copula model.

Theorem 11. Let $d \in \mathbb{N}^{*}$ and $I \in \mathbb{N}$. For every $k \in\{1, \ldots, K\}$, define

$$
a_{k}=-\frac{\sqrt{1-\left\|\rho_{k}\right\|^{2}}}{\left\|\rho_{k}\right\|}, \quad b_{k}=-\frac{\Phi\left(p_{k}\right)}{\left\|\rho_{k}\right\|},
$$

and set for every multi-indices $\mathbf{i}=\left(i_{1}, \ldots, i_{d}\right), \mathbf{j}=\left(j_{1}, \ldots, j_{d}\right) \in\{0, \ldots I\}^{d}$,

$$
\varepsilon_{K, \mathbf{i}}=\sum_{k=1}^{K} l_{k}\left(\frac{\boldsymbol{\rho}_{k}}{\left\|\boldsymbol{\rho}_{k}\right\|}\right)^{\mathbf{i}}\left(\begin{array}{c}
|\mathbf{i}| \\
\mathbf{i}
\end{array}\right) \alpha_{|\mathbf{i}|}\left(a_{k} \varepsilon_{k}+b_{k}\right)
$$

where $\alpha($.$) has been defined in Proposition 2. Then, in the d-factor model, the loss \mathcal{L}$ and $\mathcal{L}_{I}$ write as :

$$
\mathcal{L}=\sum_{i=0}^{\infty} \sum_{|\mathbf{i}|=i} \varepsilon_{K, \mathbf{i}} \mathrm{He}_{i_{1}}\left(Z_{1}\right) \ldots \mathrm{He}_{i_{d}}\left(Z_{d}\right), \quad \mathcal{L}_{I}=\sum_{i=0}^{I} \sum_{|\mathbf{i}|=i} \varepsilon_{K, \mathbf{i}} \operatorname{He}_{i_{1}}\left(Z_{1}\right) \ldots \operatorname{He}_{i_{d}}\left(Z_{d}\right)
$$


The $L_{2}$ error for the I-truncated chaos decomposition is the same as in Theorem 6 i.e. there exists $C>0$,

$$
\mathbb{E}\left[\left|\mathcal{L}-\mathcal{L}_{I}\right|^{2}\right] \leq \frac{C}{\sqrt{I}}\left(\sum_{k=1}^{K} l_{k}\right)^{2} .
$$

Before giving the proof, note that an extension to the CLT (2.27) holds also in the $d$-factor Gaussian copula model. For every multi-indices $\mathbf{i}=\left(i_{1}, \ldots, i_{d}\right), \mathbf{j}=\left(j_{1}, \ldots, j_{d}\right) \in\{0, \ldots I\}^{d}$, let

$$
\begin{gathered}
m_{K, \mathbf{i}}=\mathbb{E}\left[\varepsilon_{K, \mathbf{i}}\right]=\sum_{k=1}^{K} l_{k}\left(\frac{\boldsymbol{\rho}_{k}}{\left\|\boldsymbol{\rho}_{k}\right\|}\right)^{\mathbf{i}}\left(\begin{array}{c}
|\mathbf{i}| \\
\mathbf{i}
\end{array}\right) \mu_{|\mathbf{i}|}\left(a_{k}, b_{k}\right), \\
s_{K, \mathbf{i}, \mathbf{j}}=\operatorname{Cov}\left(\varepsilon_{K, \mathbf{i}}, \varepsilon_{K, \mathbf{j}}\right)=\sum_{k=1}^{K} l_{k}^{2}\left(\frac{\boldsymbol{\rho}_{k}}{\left\|\boldsymbol{\rho}_{k}\right\|}\right)^{\mathbf{i}+\mathbf{j}}\left(\begin{array}{c}
\mathbf{i} \mid \\
\mathbf{i}
\end{array}\right)\left(\begin{array}{c}
\mathbf{j} \mid \\
\mathbf{j}
\end{array}\right) \sigma_{|\mathbf{i}|,|\mathbf{j}|}\left(a_{k}, b_{k}\right),
\end{gathered}
$$

and define the associated mean and covariance matrix :

$$
m_{K}=\left(m_{K, \mathbf{i}}:|\mathbf{i}|=i ; i \in\{0, \ldots, I\}\right), \quad s_{K}=\left(s_{K, \mathbf{i}, \mathbf{j}}:|\mathbf{i}|=i ;|\mathbf{j}|=j ; i, j \in\{0, \ldots, I\}\right) .
$$

Under the same assumption (2.26), it is natural to approximate the vector

$$
\varepsilon_{K}=\left(\varepsilon_{K, \mathbf{i}}:|\mathbf{i}|=i ; i \in\{0, \ldots, I\}\right)
$$

with a Gaussian vector $\varepsilon_{K}^{\mathrm{G}}$ with mean $m_{K}$ and covariance matrix $s_{K}$.

Proof of Theorem 11. Remembering that $c_{k}=-\Phi^{-1}\left(p_{k}\right)$, in the $d$-multi-factor model the default event rewrites as :

$$
\left\{X_{k} \geq c_{k}\right\}=\left\{\boldsymbol{\rho}_{k} \cdot \boldsymbol{Z}+\sqrt{1-\left\|\boldsymbol{\rho}_{k}\right\|^{2}} \varepsilon_{k} \geq c_{k}\right\}=\left\{\frac{\boldsymbol{\rho}_{k}}{\left\|\boldsymbol{\rho}_{k}\right\|} \cdot \boldsymbol{Z} \geq a_{k} \varepsilon_{k}+b_{k}\right\}
$$

where $\frac{\boldsymbol{\rho}_{k}}{\left\|\boldsymbol{\rho}_{k}\right\|} \cdot \boldsymbol{Z}=\sum_{j=1}^{d} \frac{\rho_{k j}}{\sqrt{\rho_{k 1}^{2}+\cdots+\rho_{k j}^{2}}} Z_{j} \stackrel{d}{=} \mathcal{N}(0,1)$ since the $\left(Z_{j}\right)_{j=1, \ldots, d}$ are i.i.d. standard normal. We can thus apply Proposition 2 with $c=a_{k} \varepsilon_{k}+b_{k}$ and $Z=\frac{\boldsymbol{\rho}_{k}}{\left\|\boldsymbol{\rho}_{k}\right\|} \cdot \boldsymbol{Z}$ so that :

$$
\mathbb{1}_{X_{k} \geq c_{k}}=\sum_{i=0}^{\infty} \alpha_{i}\left(a_{k} \varepsilon_{k}+b_{k}\right) \operatorname{He}_{i}\left(\frac{\boldsymbol{\rho}_{k}}{\left\|\boldsymbol{\rho}_{k}\right\|} \cdot \boldsymbol{Z}\right)
$$

at any $\boldsymbol{Z}$ such that $\frac{\boldsymbol{\rho}_{k}}{\left\|\boldsymbol{\rho}_{k}\right\|} \cdot \boldsymbol{Z} \neq a_{k} \varepsilon_{k}+b_{k}$. We now need the following Lemma to proceed with the expansion of the term $\operatorname{He}_{i}\left(\frac{\boldsymbol{\rho}_{k}}{\left\|\boldsymbol{\rho}_{k}\right\|} \cdot \boldsymbol{Z}\right)$.

Lemma 12. Let $d \in \mathbb{N}^{*}, i \in \mathbb{N}$ and $\mathbf{a}=\left(a_{1}, \ldots, a_{d}\right), \mathbf{b}=\left(b_{1}, \ldots, b_{d}\right) \in \mathbb{R}^{d}$ with $\|\mathbf{a}\|=1$. Then, the following decompositon holds :

$$
\operatorname{He}_{i}(\mathbf{a} \cdot \mathbf{b})=\sum_{|\mathbf{i}|=i} \mathbf{a}^{\mathbf{i}}\left(\begin{array}{c}
i \\
\mathbf{i}
\end{array}\right) \operatorname{He}_{i_{1}}\left(b_{1}\right) \ldots \operatorname{He}_{i_{d}}\left(b_{d}\right)
$$

Proof. Taking $x=\mathbf{a} \cdot \mathbf{b}$ in (2.14), we first get :

$$
e^{\mathbf{a} \cdot \mathbf{b} w-\frac{w^{2}}{2}}=\sum_{i=0}^{\infty} \mathrm{He}_{i}(\mathbf{a} \cdot \mathbf{b}) \frac{w^{i}}{i !} .
$$

Since $\|\mathbf{a}\|=1$, notice that the exponential rewrites also as :

$$
e^{\mathbf{a} \cdot \mathbf{b} w-\frac{w^{2}}{2}}=\prod_{j=1}^{d} e^{b_{j} a_{j} w-\frac{\left(a_{j} w\right)^{2}}{2}}=\prod_{j=1}^{d} \sum_{i_{j}=0}^{\infty} \operatorname{He}_{i_{j}}\left(b_{j}\right) a_{j}^{i_{j}} \frac{w^{i_{j}}}{i_{j} !},
$$

where we have applied (2.14) with $w$ as $a_{j} w$ and $x$ as $b_{j}$. We identify each term of both series and obtain the announced claim by successively taking the derivative w.r.t. $w$ and setting $w=0$. 
Applying Lemma 12 with $\mathbf{a}=\frac{\boldsymbol{\rho}_{k}}{\left\|\boldsymbol{\rho}_{k}\right\|}$ and $\mathbf{b}=\mathbf{Z}$, we obtain :

$$
\mathbb{1}_{X_{k} \geq c_{k}}=\sum_{i=0}^{\infty} \alpha_{i}\left(a_{k} \varepsilon_{k}+b_{k}\right) \sum_{|\mathbf{i}|=i}\left(\frac{\boldsymbol{\rho}_{k}}{\left\|\boldsymbol{\rho}_{k}\right\|}\right)^{\mathbf{i}}\left(\begin{array}{c}
i \\
\mathbf{i}
\end{array}\right) \mathrm{He}_{i_{1}}\left(Z_{1}\right) \ldots \mathrm{He}_{i_{d}}\left(Z_{d}\right),
$$

and this gives (3.3).

The estimate (3.4) remains the same as in Theorem 6 noticing that $\mathcal{L}=\sum_{i=0}^{\infty} \alpha_{i}\left(a_{k} \varepsilon_{k}+b_{k}\right) \mathrm{He}_{i}\left(\frac{\boldsymbol{\rho}_{k}}{\left\|\boldsymbol{\rho}_{k}\right\|} \cdot \boldsymbol{Z}\right)$ with $\frac{\boldsymbol{\rho}_{k}}{\left\|\boldsymbol{\rho}_{k}\right\|} \cdot \boldsymbol{Z} \stackrel{d}{=} \mathcal{N}(0,1)$, because in the proof of Theorem 6 , we only need that $\left(\varepsilon_{k}\right)_{1 \leq k \leq K}$ is independent of $Z$.

\section{Conclusion}

In this paper, through the use of the Wiener chaos decomposition we have designed a meta-model to significantly reduce the computational effort needed to sample from the loss distribution of very large credit portfolio. Instead of going over all $\mathrm{K}$ obligors, we essentially sum over a $I+1$ Gaussian vector whose characteristics can be computed off-line. Our approach gives accurate and fast results that have been numerically tested on two different portfolios. We have seen that even with a small $I$, the approximation performs well even in the tails (up to $99.99 \%$ quantile). Such method can therefore be used to estimate risk measures such as VaR and CVaR that need to be computed often for regulatory purposes. The next step would now be to give an estimate of the Gaussian approximation error between $\mathcal{L}_{I}$ and $\mathcal{L}_{I}^{\mathrm{G}}$ so that we could get an estimate of the full error between $\mathcal{L}$ and $\mathcal{L}_{I}^{\mathrm{G}}$. This is a difficult issue, since the Gaussian dimension is asymptotically infinite (as $I \rightarrow+\infty)$, and we will tackle this analysis in future research.

\section{Technical proofs}

\subsection{Proof of Proposition 2}

By definition $\alpha_{i}(c)=\frac{1}{i !} \mathbb{E}\left[\mathbb{1}_{c \leq Z} \mathrm{He}_{i}(Z)\right]$. Hence :

$$
\alpha_{0}(c)=\mathbb{P}(Z \geq c)=\Phi(-c)
$$

and using Equation (2.4), we have for every $i \in \mathbb{N}^{*}$ :

$$
\begin{aligned}
\alpha_{i}(c)=\frac{1}{i !} \int_{c}^{\infty} \mathrm{He}_{i}(Z) \frac{e^{-\frac{z^{2}}{2}}}{\sqrt{2 \pi}} \mathrm{d} z=\frac{(-1)^{i}}{i ! \sqrt{2 \pi}} \int_{c}^{\infty} \frac{\mathrm{d}^{i}}{\mathrm{~d} z^{i}}\left(e^{-\frac{z^{2}}{2}}\right) \mathrm{d} z & =\left.\frac{(-1)^{i-1}}{i ! \sqrt{2 \pi}} \frac{\mathrm{d}^{i-1}}{\mathrm{~d} z^{i-1}}\left(e^{-\frac{z^{2}}{2}}\right)\right|_{z=c} \\
& =\frac{e^{-\frac{c^{2}}{2}} \operatorname{He}_{i-1}(c)}{i ! \sqrt{2 \pi}} .
\end{aligned}
$$

Consequently $\alpha_{1}(c)=\frac{e^{-\frac{c^{2}}{2}}}{\sqrt{2 \pi}}$ and using Equation (2.5), one has :

$$
\alpha_{i+2}(c)=\frac{e^{-\frac{c^{2}}{2}} \mathrm{He}_{i+1}(c)}{(i+2) ! \sqrt{2 \pi}}=\frac{e^{-\frac{c^{2}}{2}}}{(i+2) ! \sqrt{2 \pi}}\left(c \mathrm{He}_{i}(c)-i \mathrm{He}_{i-1}(c)\right)=\frac{c}{i+2} \alpha_{i+1}(c)-\frac{i}{(i+1)(i+2)} \alpha_{i}(c) .
$$

Finally, the explicit expression for $\alpha$ is a direct consequence of the relation $\alpha_{i}(c)=\frac{e^{-\frac{c^{2}}{2}} \mathrm{He}_{i-1}(c)}{i ! \sqrt{2 \pi}}$ and Equation $(2.7)$. 


\subsection{Proof of Lemma 4}

We proceed as in [Sze75, Theorem 7.6.1]. Using Equation (2.10), a direct computation shows that the function $z(x):=\operatorname{He}_{n}(x) e^{-\frac{x^{2}}{2}}$ solves the following ordinary differential equation (ODE):

$$
z^{\prime \prime}(x)+x z^{\prime}(x)+(n+1) z(x)=0
$$

Now let $(n+1) f(x):=(n+1)\left(\operatorname{He}_{n}(x) e^{-\frac{x^{2}}{2}}\right)^{2}+\left(\frac{\mathrm{d}}{\mathrm{d} x}\left\{\operatorname{He}_{n}(x) e^{-\frac{x^{2}}{2}}\right\}\right)^{2}$ and notice that $f(x)=\left(\operatorname{He}_{n}(x) e^{-\frac{x^{2}}{2}}\right)^{2}$ when $\left(\frac{\mathrm{d}}{\mathrm{d} x}\left\{\operatorname{He}_{n}(x) e^{-\frac{x^{2}}{2}}\right\}\right)^{2}=0$. Further using the ODE above, we have :

$$
\begin{aligned}
(n+1) f^{\prime}(x) & =2(n+1)\left(\operatorname{He}_{n}(x) e^{-\frac{x^{2}}{2}}\right) \frac{\mathrm{d}}{\mathrm{d} x}\left\{\operatorname{He}_{n}(x) e^{-\frac{x^{2}}{2}}\right\}+2 \frac{\mathrm{d}}{\mathrm{d} x}\left\{\operatorname{He}_{n}(x) e^{-\frac{x^{2}}{2}}\right\} \frac{\mathrm{d}^{2}}{\mathrm{~d} x^{2}}\left\{\operatorname{He}_{n}(x) e^{-\frac{x^{2}}{2}}\right\} \\
& =-2 x\left(\frac{\mathrm{d}}{\mathrm{d} x}\left\{\operatorname{He}_{n}(x) e^{-\frac{x^{2}}{2}}\right\}\right)^{2} .
\end{aligned}
$$

For $x>0$ (resp. $x<0$ ) the derivative of $f$ is strictly negative (resp. positive) except at a finite number of points. Hence $f(x)$ is (strictly) decreasing on $x \geq 0$ (resp. increasing on $x \leq 0$ ). Remind that at the points of maxima of $x \mapsto z^{2}(x), f(x)$ and $z^{2}(x)$ coincide, whence the announced result.

\subsection{Proof of Corollary 5}

Let us define $z_{n}: x \in \mathbb{R} \rightarrow \frac{e^{-\frac{x^{2}}{2}} \mathrm{He}_{n}(x)}{\sqrt{(n+1) !}}$ and notice that $z_{n}^{2}$ is an even function owing to (2.6). We thus restrict our attention to $x \geq 0$. It is known that zeros of orthogonal polynomials are both real and distinct (see [Sze75, Theorem 3.3.1]): therefore, for any $m \in \mathbb{N}^{*}$ we can denote the $2 m$ (resp. $\left.2 m-1\right)$ zeros of He $2 m(x)$ (resp. $\left.\mathrm{He}_{2 m-1}(x)\right)$ by $x_{-m, 2 m}<\cdots<x_{-1,2 m}<0<x_{1,2 m}<\cdots<x_{m, 2 m}$ (resp. $x_{-(m-1), 2 m-1}<\cdots<$ $\left.x_{0,2 m-1}=0<\cdots<x_{m-1,2 m-1}\right)$. Also, the derivative of $z_{n}^{2}$ is given by :

$$
\begin{gathered}
\left(z_{n}^{2}(x)\right)^{\prime} \stackrel{(2.11)}{=} \frac{1}{(n+1) !}\left(-2 x e^{-x^{2}} \operatorname{He}_{n}^{2}(x)+2 e^{-x^{2}} n \mathrm{He}_{n}(x) \mathrm{He}_{n-1}(x)\right) \\
=\frac{2 e^{-x^{2}} \operatorname{He}_{n}(x)}{(n+1) !}\left(-x \operatorname{He}_{n}(x)+n \operatorname{He}_{n-1}(x)\right) \\
\stackrel{(2.5)}{=}-\frac{2 e^{-x^{2}} \operatorname{He}_{n}(x) \operatorname{He}_{n+1}(x)}{(n+1) !}
\end{gathered}
$$

Therefore, the critical points of $z_{n}^{2}$ are the zeros of $\mathrm{He}_{n}$ and $\mathrm{He}_{n+1}$. But $z_{n}^{2}$ is non-negative, vanishes at the $n$ zeros of $\mathrm{He}_{n}$, and converges to 0 at $\pm \infty$; therefore the points of maxima of $z_{n}^{2}$ must be the $n+1$ zeros of $\mathrm{He}_{n+1}$. By Lemma 4, the maxima of $z_{n}^{2}$ is achieved at $x_{0, n}=0$ (resp. $x_{1, n}$ ) if $n$ is odd (resp. even). In view of (2.9), we obtain for any $n \in \mathbb{N}$,

$$
M_{n}= \begin{cases}\frac{\mathrm{He}_{2 m}^{2}(0)}{(2 m+1) !}=\frac{(2 m) !}{(2 m+1)(m !)^{2} 2^{2 m}}, & \text { if } n=2 m \\ \frac{e^{-\left(x_{1,2 m+2}\right)^{2}} \mathrm{He}_{2 m+1}^{2}\left(x_{1,2 m+2}\right)}{(2 m+2) !}, & \text { if } n=2 m+1 .\end{cases}
$$

Let us now prove that $M_{2 m+1}<M_{2 m}$ for every $m \in \mathbb{N}$. First notice that the following recursive relations for $z_{n}$ hold:

$$
\begin{aligned}
z_{n+2}(x) & =\frac{\operatorname{He}_{n+2}(x) e^{-\frac{x^{2}}{2}}}{\sqrt{(n+3) !}} \\
& \stackrel{(2.5)}{=} \frac{\left(x \operatorname{He}_{n+1}(x)-(n+1) \mathrm{He}_{n}(x)\right) e^{-\frac{x^{2}}{2}}}{\sqrt{(n+3) !}}
\end{aligned}
$$




$$
\begin{aligned}
& =\frac{x \mathrm{He}_{n+1}(x) e^{-\frac{x^{2}}{2}}}{\sqrt{n+3} \sqrt{(n+2) !}}-\frac{(n+1) \mathrm{He}_{n}(x) e^{-\frac{x^{2}}{2}}}{\sqrt{(n+3)(n+2)} \sqrt{(n+1) !}} \\
& =\frac{x}{\sqrt{n+3}} z_{n+1}(x)-\frac{n+1}{\sqrt{(n+3)(n+2)}} z_{n}(x), \\
z_{n}^{\prime}(x) & \stackrel{(2.11)}{=} \frac{\left(n \mathrm{He}_{n-1}(x)-x \mathrm{He}_{n}(x)\right) e^{-\frac{x^{2}}{2}}}{\sqrt{(n+1) !}} \\
z_{n}^{\prime \prime}(x) & \stackrel{\left(\text { (2.5) }^{\prime}\right.}{=}-\sqrt{n+2)} z_{n+1}(x), \\
& \stackrel{(5.4)}{=} \sqrt{(n+2)} z_{n+1}^{\prime}(x) \\
& \stackrel{(5.3)}{=} x \sqrt{n+2} z_{n+1}(x)-(n+1) z_{n}(x) \\
& \stackrel{(5.4)}{=}-x z_{n}^{\prime}(x)-(n+1) z_{n}(x) .
\end{aligned}
$$

Now define the map $f_{n}: x \rightarrow\left(z_{n}(x)\right)^{2}+\frac{1}{n+1}\left(z_{n}^{\prime}(x)\right)^{2} \stackrel{(5.4)}{=}\left(z_{n}(x)\right)^{2}+\frac{n+2}{n+1}\left(z_{n+1}(x)\right)^{2}$ whose derivative equals:

$$
f_{n}^{\prime}(x)=2 z_{n}^{\prime}(x)\left(z_{n}(x)+\frac{z_{n}^{\prime \prime}(x)}{n+1}\right) \stackrel{(5.5)}{=}-\frac{2 x}{n+1}\left(z_{n}^{\prime}(x)\right)^{2} .
$$

Hence $f_{n}$ is (strictly) decreasing on $[0,+\infty)\left(z_{n}^{\prime}\right.$ vanishes only at a finite number of points). Now consider the case where $n$ is even, i.e $n=2 m$ for $m \in \mathbb{N}$; then using that $x_{1,2 m+2}>0$ we have :

$$
f_{2 m}\left(x_{1,2 m+2}\right)<f_{2 m}(0) .
$$

On the other hand, $f_{2 m}(0)=\left(z_{2 m}(0)\right)^{2}+\frac{2 m+2}{2 m+1}\left(z_{2 m+1}(0)\right)^{2} \stackrel{(2.9)}{=}\left(z_{2 m}(0)\right)^{2}=M_{2 m}$ and $f_{2 m}\left(x_{1,2 m+2}\right)=$ $\left(z_{2 m}\left(x_{1,2 m+2}\right)\right)^{2}+\frac{2 m+2}{2 m+1}\left(z_{2 m+1}\left(x_{1,2 m+2}\right)\right)^{2}>M_{2 m+1}$. Finally, owing to (5.6) we get :

$$
M_{2 m+1}<M_{2 m}
$$

which concludes the proof.

\subsection{Proof of Proposition 8}

In the proof, $X, Y, Z$ are independent standard normal random variables.

$\triangleright$ Results on the mean $\mu$.(.,.). By definition of $\alpha_{0}$ (see (5.1)) we get:

$$
\mu_{0}(a, b)=\mathbb{E}[\Phi(-a X-b)]=\mathbb{E}[\mathbb{P}(Y<-a X-b \mid X)] .
$$

Using that $a X+Y \stackrel{d}{=} \mathcal{N}\left(0,1+a^{2}\right)$, we get :

$$
\mathbb{E}\left[\alpha_{0}(a X+b)\right]=\Phi\left(-\frac{b}{\sqrt{1+a^{2}}}\right) .
$$

Now we compute $\mu_{1}(a, b)$ : invoke the expression of $\alpha_{1}(\cdot)$ from (5.2) to write

$$
\mu_{1}(a, b)=\int_{\mathbb{R}} \frac{e^{-\frac{(a X+b)^{2}}{2}}}{\sqrt{2 \pi}} \frac{e^{-\frac{x^{2}}{2}}}{\sqrt{2 \pi}} \mathrm{d} x=\frac{e^{-\frac{b^{2}}{2\left(1+a^{2}\right)}}}{\sqrt{2 \pi}} \int_{\mathbb{R}} \frac{e^{-\frac{1+a^{2}}{2}\left(x+\frac{a b}{1+a^{2}}\right)^{2}}}{\sqrt{2 \pi}} \mathrm{d} x=\frac{e^{-\frac{b^{2}}{2\left(1+a^{2}\right)}}}{\sqrt{2 \pi} \sqrt{1+a^{2}}} .
$$

We turn to the derivation of the three-term recurrence relation for $\mu_{i}(a, b)$. From Proposition 2, it follows that

$$
\mu_{i+2}(a, b)=\mathbb{E}\left[\frac{a X+b}{i+2} \alpha_{i+1}(a X+b)-\frac{i}{(i+1)(i+2)} \alpha_{i}(a X+b)\right]
$$




$$
=\frac{a}{i+2} \mathbb{E}\left[X \alpha_{i+1}(a X+b)\right]+\frac{b}{i+2} \mu_{i+1}(a, b)-\frac{i}{(i+1)(i+2)} \mu_{i}(a, b) .
$$

An integration by parts gives

$$
\begin{aligned}
\mathbb{E}\left[X \alpha_{i+1}(a X+b)\right] & =\left.\frac{(-1)^{i}}{(i+1) ! \sqrt{2 \pi}} \int_{\mathbb{R}} x \frac{\mathrm{d}^{i}}{\mathrm{~d} z^{i}}\left(e^{-\frac{z^{2}}{2}}\right)\right|_{z=a x+b} \frac{e^{-\frac{x^{2}}{2}}}{\sqrt{2 \pi}} \mathrm{d} x \\
& =\left.\frac{(-1)^{i}}{(i+1) ! \sqrt{2 \pi}} a \int_{\mathbb{R}} \frac{\mathrm{d}^{i+1}}{\mathrm{~d} z^{i+1}}\left(e^{-\frac{z^{2}}{2}}\right)\right|_{z=a x+b} \frac{e^{-\frac{x^{2}}{2}}}{\sqrt{2 \pi}} \mathrm{d} x \\
& =-a(i+2) \mu_{i+2}(a, b) .
\end{aligned}
$$

Plugging the above into (5.7) readily gives

$$
\left(1+a^{2}\right) \mu_{i+2}(a, b)=\frac{b}{i+2} \mu_{i+1}(a, b)-\frac{i}{(i+1)(i+2)} \mu_{i}(a, b) .
$$

$\triangleright$ Initialization of the variance/covariance terms. First, we have :

$$
\sigma_{0,0}(a, b)=\mathbb{E}\left[\Phi(-a X-b)^{2}\right]-\mathbb{E}[\Phi(-a X-b)]^{2} .
$$

Now notice that :

$$
\begin{aligned}
\mathbb{E}\left[\Phi(-a X-b)^{2}\right] & =\int_{\mathbb{R}}\left(\int_{\mathbb{R}^{2}} \mathbb{1}_{y<-a x-b} \frac{e^{-\frac{y^{2}}{2}}}{\sqrt{2 \pi}} \mathrm{d} y \mathbb{1}_{z<-a x-b} \frac{e^{-\frac{z^{2}}{2}}}{\sqrt{2 \pi}} \mathrm{d} z\right) \frac{e^{-\frac{x^{2}}{2}}}{\sqrt{2 \pi}} \mathrm{d} x \\
& =\mathbb{E}\left[\mathbb{1}_{Y+a X<-b} \mathbb{1}_{Z+a X<-b}\right]
\end{aligned}
$$

using that $X, Y, Z$ are i.i.d. Whence, since $Y+a X \stackrel{d}{=} \mathcal{N}\left(0,1+a^{2}\right), Z+a X \stackrel{d}{=} \mathcal{N}\left(0,1+a^{2}\right)$ and $\operatorname{Cov}(Y+a X, Z+a X)=a^{2}$, we get

$$
\mathbb{E}\left[\Phi(-a X-b)^{2}\right]=\Phi_{\Sigma}\left((-b,-b)^{\top}\right)
$$

where $\Sigma=\left(\begin{array}{cc}1+a^{2} & a^{2} \\ a^{2} & 1+a^{2}\end{array}\right)$.

Consider now the covariance. For the sake of clarity we introduce the notation

$$
\rho_{i, j}(a, b):=\mathbb{E}\left[\alpha_{i}(a X+b) \alpha_{j}(a X+b)\right] \Longleftrightarrow \sigma_{i, j}(a, b)=\rho_{i, j}(a, b)-\mu_{i}(a, b) \mu_{j}(a, b) .
$$

Then we have :

$$
\begin{aligned}
\rho_{0,1}(a, b) & =\mathbb{E}\left[\alpha_{0}(a X+b) \alpha_{1}(a X+b)\right] \\
& =\int_{\mathbb{R}} \alpha_{0}(a X+b) \alpha_{1}(a X+b) \frac{e^{-\frac{x^{2}}{2}}}{\sqrt{2 \pi}} \mathrm{d} x \\
& =\int_{\mathbb{R}} \Phi(-a x-b) \frac{e^{-\frac{(a X+b)^{2}}{2}}}{\sqrt{2 \pi}} \frac{e^{-\frac{x^{2}}{2}}}{\sqrt{2 \pi}} \mathrm{d} x \\
& =\frac{e^{-\frac{b^{2}}{2\left(1+a^{2}\right)}}}{\sqrt{2 \pi}} \int_{\mathbb{R}} \Phi(-a x-b) \frac{e^{-\frac{1+a^{2}}{2}}\left(x+\frac{a b}{1+a^{2}}\right)^{2}}{\sqrt{2 \pi}} \mathrm{d} x
\end{aligned}
$$

(setting $\left.y=\sqrt{1+a^{2}} x+\frac{a b}{\sqrt{1+a^{2}}}\right)$

$$
=\frac{e^{-\frac{b^{2}}{2\left(1+a^{2}\right)}}}{\sqrt{2 \pi} \sqrt{1+a^{2}}} \int_{\mathbb{R}} \Phi\left(-\frac{a}{\sqrt{1+a^{2}}} y-\frac{b}{1+a^{2}}\right) \frac{e^{-\frac{y^{2}}{2}}}{\sqrt{2 \pi}} \mathrm{d} y
$$




$$
\begin{aligned}
& =\frac{e^{-\frac{b^{2}}{2\left(1+a^{2}\right)}}}{\sqrt{2 \pi} \sqrt{1+a^{2}}} \mu_{0}\left(\frac{a}{\sqrt{1+a^{2}}}, \frac{b}{1+a^{2}}\right) \\
& =\mu_{1}(a, b) \mu_{0}\left(\frac{a}{\sqrt{1+a^{2}}}, \frac{b}{1+a^{2}}\right) .
\end{aligned}
$$

We get the announced result for $\sigma_{0,1}(a, b)$ owing to (5.8).

$\triangleright$ Three-term recurrence relation on variance/covariance. Similarly, using (2.17) in Proposition 2 yields

$$
\begin{aligned}
\rho_{0, i+2}(a, b) & =\mathbb{E}\left[\alpha_{0}(a X+b) \alpha_{i+2}(a X+b)\right] \\
& =\frac{a}{i+2} \mathbb{E}\left[X \alpha_{0}(a X+b) \alpha_{i+1}(a X+b)\right]+\frac{b}{i+2} \rho_{0, i+1}(a, b)-\frac{i}{(i+1)(i+2)} \rho_{0, i}(a, b) .
\end{aligned}
$$

Integrating by parts as before yields :

$$
\begin{aligned}
\mathbb{E}\left[X \alpha_{0}(a X+b) \alpha_{i+1}(a X+b)\right]= & \left.\frac{(-1)^{i}}{(i+1) ! \sqrt{2 \pi}} \int_{\mathbb{R}} x \Phi(-a x-b) \frac{\mathrm{d}^{i}}{\mathrm{~d} z^{i}}\left(e^{-\frac{z^{2}}{2}}\right)\right|_{z=a x+b} \frac{e^{-\frac{x^{2}}{2}}}{\sqrt{2 \pi}} \mathrm{d} x \\
= & \frac{(-1)^{i}}{(i+1) ! \sqrt{2 \pi}} \int_{\mathbb{R}}\left(-\left.a \frac{e^{-\frac{(a X+b)^{2}}{2}}}{\sqrt{2 \pi}} \frac{\mathrm{d}^{i}}{\mathrm{~d} z^{i}}\left(e^{-\frac{z^{2}}{2}}\right)\right|_{z=a x+b}\right. \\
& \left.+\left.a \Phi(-a x-b) \frac{\mathrm{d}^{i+1}}{\mathrm{~d} z^{i+1}}\left(e^{-\frac{z^{2}}{2}}\right)\right|_{z=a x+b}\right) \frac{e^{-\frac{x^{2}}{2}}}{\sqrt{2 \pi}} \mathrm{d} x .
\end{aligned}
$$

Using the new variable $y=\sqrt{1+a^{2}} x+\frac{a b}{\sqrt{1+a^{2}}}$, we show (similar computations to (5.9)) that the first integral is equal to

$$
\begin{aligned}
& -\left.\frac{a(-1)^{i} e^{-\frac{b^{2}}{2\left(1+a^{2}\right)}}}{(i+1) ! \sqrt{2 \pi} \sqrt{1+a^{2}}} \int_{\mathbb{R}} \frac{1}{\sqrt{2 \pi}} \frac{\mathrm{d}^{i}}{\mathrm{~d} z^{i}}\left(e^{-\frac{z^{2}}{2}}\right)\right|_{z=\frac{a}{\sqrt{1+a^{2}}} y+\frac{b}{1+a^{2}}} \frac{e^{-\frac{y^{2}}{2}}}{\sqrt{2 \pi}} \mathrm{d} y \\
= & -a \mu_{1}(a, b) \mu_{i+1}\left(\frac{a}{\sqrt{1+a^{2}}}, \frac{b}{1+a^{2}}\right) .
\end{aligned}
$$

Besides, the second integral at the right-hand side of (5.10) is

$$
\begin{aligned}
& -\left.\frac{a(-1)^{i+1}(i+2)}{(i+2) ! \sqrt{2 \pi}} \int_{\mathbb{R}} \Phi(-a x-b) \frac{\mathrm{d}^{i+1}}{\mathrm{~d} z^{i+1}}\left(e^{-\frac{z^{2}}{2}}\right)\right|_{z=a x+b} \frac{e^{-\frac{x^{2}}{2}}}{\sqrt{2 \pi}} \mathrm{d} x \\
= & -a(i+2) \rho_{0, i+2}(a, b) .
\end{aligned}
$$

All in all, (5.10) becomes finally :

$$
\left(1+a^{2}\right) \rho_{0, i+2}(a, b)=-\frac{a^{2}}{i+2} \mu_{1}(a, b) \mu_{i+1}\left(\frac{a}{\sqrt{1+a^{2}}}, \frac{b}{1+a^{2}}\right)+\frac{b}{i+2} \rho_{0, i+1}(a, b)-\frac{i}{(i+1)(i+2)} \rho_{0, i}(a, b) ;
$$

owing to (5.8), and by substracting $\left(1+a^{2}\right) \mu_{0}(a, b)$ times the recursive relation (3.1) on $\mu$. $(a, b)$, the above is equivalent to

$$
\left(1+a^{2}\right) \sigma_{0, i+2}(a, b)=-\frac{a^{2}}{i+2} \mu_{1}(a, b) \mu_{i+1}\left(\frac{a}{\sqrt{1+a^{2}}}, \frac{b}{1+a^{2}}\right)+\frac{b}{i+2} \sigma_{0, i+1}(a, b)-\frac{i}{(i+1)(i+2)} \sigma_{0, i}(a, b) .
$$

The proof of iterative equation (3.2) for $\sigma_{0, .}(a, b)$ is complete.

We now handle the proof for the relation on $\sigma_{i, j}(a, b)$ where $i, j \in \mathbb{N}$. We proceed analogously; starting from (2.17), write

$$
\rho_{i, j+2}(a, b)=\mathbb{E}\left[\alpha_{i}(a X+b) \alpha_{j+2}(a X+b)\right]
$$




$$
=\frac{a}{j+2} \mathbb{E}\left[X \alpha_{i}(a X+b) \alpha_{j+1}(a X+b)\right]+\frac{b}{j+2} \rho_{i, j+1}(a, b)-\frac{j}{(j+1)(j+2)} \rho_{i, j}(a, b) .
$$

Next, we derive

$$
\begin{aligned}
& \mathbb{E}\left[X \alpha_{i}(a X+b) \alpha_{j+1}(a X+b)\right] \\
= & \left.\left.\frac{(-1)^{i-1}}{i ! \sqrt{2 \pi}} \frac{(-1)^{j}}{(j+1) ! \sqrt{2 \pi}} \int_{\mathbb{R}} x \frac{\mathrm{d}^{i-1}}{\mathrm{~d} z^{i-1}}\left(e^{-\frac{z^{2}}{2}}\right)\right|_{z=a x+b} \frac{\mathrm{d}^{j}}{\mathrm{~d} z^{j}}\left(e^{-\frac{z^{2}}{2}}\right)\right|_{z=a x+b} \frac{e^{-\frac{x^{2}}{2}}}{\sqrt{2 \pi}} \mathrm{d} x \\
= & \frac{(-1)^{i-1}}{i ! \sqrt{2 \pi}} \frac{(-1)^{j} a}{(j+1) ! \sqrt{2 \pi}} \int_{\mathbb{R}}\left(\left.\left.\frac{\mathrm{d}^{i}}{\mathrm{~d} z^{i}}\left(e^{-\frac{z^{2}}{2}}\right)\right|_{z=a x+b} \frac{\mathrm{d}^{j}}{\mathrm{~d} z^{j}}\left(e^{-\frac{z^{2}}{2}}\right)\right|_{z=a x+b}\right. \\
& \left.\quad+\left.\left.\frac{\mathrm{d}^{i-1}}{\mathrm{~d} z^{i-1}}\left(e^{-\frac{z^{2}}{2}}\right)\right|_{z=a x+b} \frac{\mathrm{d}^{j+1}}{\mathrm{~d} z^{j+1}}\left(e^{-\frac{z^{2}}{2}}\right)\right|_{z=a x+b}\right) \frac{e^{-\frac{x^{2}}{2}}}{\sqrt{2 \pi}} \mathrm{d} x \\
= & -a\left((i+1) \rho_{i+1, j+1}(a, b)+(j+2) \rho_{i, j+2}(a, b)\right) .
\end{aligned}
$$

Therefore, all in all, we get

$$
\left(1+a^{2}\right) \rho_{i, j+2}(a, b)=-\frac{a^{2}(i+1)}{j+2} \rho_{i+1, j+1}(a, b)+\frac{b}{j+2} \rho_{i, j+1}(a, b)-\frac{j}{(j+1)(j+2)} \rho_{i, j}(a, b) .
$$

Accounting for (2.17), we obtain

$$
\left(1+a^{2}\right) \sigma_{i, j+2}(a, b)=-\frac{a^{2}(i+1)}{j+2} \rho_{i+1, j+1}(a, b)+\frac{b}{j+2} \sigma_{i, j+1}(a, b)-\frac{j}{(j+1)(j+2)} \sigma_{i, j}(a, b) .
$$

Now, express $\rho_{i+1, j+1}(a, b)$ as a function of the other terms and this gives the announced result in (3.2).

\subsection{Proof of Lemma 9}

Let $A, B \in \mathbb{R}$ and $X \stackrel{d}{=} \mathcal{N}(0,1)$. It is well-known that the moments of the standard normal distribution are given by :

$$
\mathbb{E}\left[X^{m}\right]=\int_{\mathbb{R}} x^{m} \frac{e^{-\frac{x^{2}}{2}}}{\sqrt{2 \pi}} \mathrm{d} x=\left\{\begin{array}{ll}
0 & \text { if } m=2 k+1,(k \in \mathbb{N}) \\
\frac{(2 k) !}{2^{k} k !} & \text { if } m=2 k,(k \in \mathbb{N})
\end{array} .\right.
$$

From the above relation, we obtain :

$$
\mathbb{E}\left[\mathrm{He}_{i}(A X+B)\right] \stackrel{(2.8)}{=} \sum_{m=0}^{i}\left(\begin{array}{c}
i \\
m
\end{array}\right) A^{m} \mathrm{He}_{i-m}(B) \int_{\mathbb{R}} x^{m} \frac{e^{-x^{2} / 2}}{\sqrt{2 \pi}} \mathrm{d} x \stackrel{(5.11)}{=} \sum_{k=0}^{\left\lfloor\frac{i}{2}\right\rfloor} \frac{i ! A^{2 k} \mathrm{He}_{i-2 k}(B)}{2^{k} k !(i-2 k) !} .
$$

By the identity $-\frac{(a x+b)^{2}}{2}-\frac{x^{2}}{2}=-\frac{1}{2}\left(\sqrt{1+a^{2}} x+\frac{a b}{\sqrt{1+a^{2}}}\right)^{2}-\frac{b^{2}}{2\left(1+a^{2}\right)}$, we have :

$$
\begin{aligned}
\mu_{i}(a, b) & \stackrel{(2.16)}{=} \frac{1}{\sqrt{2 \pi} i !} \int_{\mathbb{R}} \operatorname{He}_{i-1}(a x+b) e^{-\frac{(a x+b)^{2}}{2}} \frac{e^{-\frac{x^{2}}{2}}}{\sqrt{2 \pi}} \mathrm{d} x \\
& =\frac{e^{-\frac{b^{2}}{2\left(1+a^{2}\right)}}}{\sqrt{2 \pi} i !} \int_{\mathbb{R}} \operatorname{He}_{i-1}(a x+b) \frac{e^{-\frac{1}{2}\left(\sqrt{1+a^{2}} x+\frac{a b}{\sqrt{1+a^{2}}}\right)^{2}}}{\sqrt{2 \pi}} \mathrm{d} x \\
& =\frac{e^{-\frac{b^{2}}{2\left(1+a^{2}\right)}}}{\sqrt{2 \pi} i ! \sqrt{1+a^{2}}} \int_{\mathbb{R}} \operatorname{He}_{i-1}\left(\frac{a}{\sqrt{1+a^{2}}} y+\frac{b}{1+a^{2}}\right) \frac{e^{-\frac{y^{2}}{2}}}{\sqrt{2 \pi}} \mathrm{d} y
\end{aligned}
$$

where we have introduced the new variable $y=\sqrt{1+a^{2}} x+\frac{a b}{\sqrt{1+a^{2}}}$ in the last equality. The expression for $\mu_{i}(a, b)$ is then a direct consequence of (5.12) with $A=\frac{a}{\sqrt{1+a^{2}}}$ and $B=\frac{b}{1+a^{2}}$. Similarly, we get :

$\mathbb{E}\left[\mathrm{He}_{i}(A X+B) \mathrm{He}_{j}(A X+B)\right]=\sum_{m=0}^{i} \sum_{n=0}^{j}\left(\begin{array}{c}i \\ m\end{array}\right)\left(\begin{array}{c}j \\ n\end{array}\right) A^{m+n} \mathrm{He}_{i-m}(B) \mathrm{He}_{j-n}(B) \int_{\mathbb{R}} x^{m+n} \frac{e^{-x^{2} / 2}}{\sqrt{2 \pi}} \mathrm{d} x$ 


$$
\begin{aligned}
& =\sum_{k=0}^{\left\lfloor\frac{i}{2}\right\rfloor} \sum_{p=0}^{\left\lfloor\frac{j}{2}\right\rfloor}\left(\begin{array}{c}
i \\
2 k
\end{array}\right)\left(\begin{array}{c}
j \\
2 p
\end{array}\right) \frac{A^{2(k+p)} \mathrm{He}_{i-2 k}(B) \mathrm{He}_{j-2 p}(B)(2(k+p)) !}{2^{k+p}(k+p) !} \\
& +\sum_{k=0}^{\left\lfloor\frac{i-1}{2}\right\rfloor} \sum_{p=0}^{\left\lfloor\frac{j-1}{2}\right\rfloor}\left(\begin{array}{c}
i \\
2 k+1
\end{array}\right)\left(\begin{array}{c}
j \\
2 p+1
\end{array}\right) \frac{A^{2(k+p+1)} \mathrm{He}_{i-2 k-1}(B) \mathrm{He}_{j-2 p-1}(B)(2(k+p+1)) !}{2^{k+p+1}(k+p+1) !} .
\end{aligned}
$$

By the identity $-(a x+b)^{2}-\frac{x^{2}}{2}=-\frac{1}{2}\left(\sqrt{1+2 a^{2}} x+\frac{2 a b}{\sqrt{1+2 a^{2}}}\right)^{2}-\frac{b^{2}}{1+2 a^{2}}$, the covariance rewrites :

$$
\begin{aligned}
\sigma_{i, j}(a, b) & =\frac{1}{2 \pi i ! j !} \int_{\mathbb{R}} \operatorname{He}_{i-1}(a x+b) \mathrm{He}_{j-1}(a x+b) e^{-(a x+b)^{2}} \frac{e^{-\frac{x^{2}}{2}}}{\sqrt{2 \pi}} \mathrm{d} x-\mu_{i}(a, b) \mu_{j}(a, b) \\
& =\frac{e^{-\frac{b^{2}}{1+2 a^{2}}}}{2 \pi i ! j ! \sqrt{1+2 a^{2}}} \int_{\mathbb{R}} \operatorname{He}_{i-1}\left(\frac{a}{\sqrt{1+2 a^{2}}} y+\frac{b}{1+2 a^{2}}\right) \mathrm{He}_{j-1}\left(\frac{a}{\sqrt{1+2 a^{2}}} y+\frac{b}{1+2 a^{2}}\right) \frac{e^{-\frac{y^{2}}{2}}}{\sqrt{2 \pi}} \mathrm{d} y \\
& -\mu_{i}(a, b) \mu_{j}(a, b),
\end{aligned}
$$

where we have introduced this time $y=\sqrt{1+2 a^{2}} x+\frac{2 a b}{\sqrt{1+2 a^{2}}}$. We conclude using (5.13) with $A=\frac{a}{\sqrt{1+2 a^{2}}}$ and $B=\frac{b}{1+2 a^{2}}$.

\section{References}

[ADEH99] P. Artzner, F. Delbaen, J.-M. Eber, and D. Heath. Coherent measures of risk. Mathematical finance, $9(3): 203-228,1999.1$

[ASB03] L. Andersen, J. Sidenius, and S. Basu. All your hedges in one basket. Risk, 16(11):67-72, 2003. 1

[Bai48] W.N. Bailey. Some integrals involving Hermite polynomials. Journal of the London Mathematical Society, s1-23(4):291-297, 1948. 2.2

[BR10] R.N. Bhattacharya and R.R. Rao. Normal Approximation and Asymptotic Expansions, volume 64. SIAM, 2010. 2.3

[BR13] T.R. Bielecki and M. Rutkowski. Credit risk: modelling, valuation and hedging. Springer-Verlag \& Business Media, 2013. 1

[CLV04] U. Cherubini, E. Luciano, and W. Vecchiato. Copula methods in finance. John Wiley \& Sons, 2004. 1

[Fun92] D. Funaro. Polynomial approximation of differential equations, volume 8 of Lecture Notes in Physics. New Series m: Monographs. Springer-Verlag, Berlin, 1992. 2.2

[GL05] P. Glasserman and J. Li. Importance sampling for portfolio credit risk. Management science, 51(11):1643-1656, 2005. 1, 7, 3.3

[JEK08] Y. Jiao, N. El Karoui, and D. Kurtz. Gaussian and Poisson approximation: applications to CDOs tranche pricing. Journal of Computational Finance, 12(2):31-58, 2008. 1

[JS13] J. Jacod and A. Shiryaev. Limit theorems for stochastic processes, volume 288. Springer Science \& Business Media, 2013. 2.3

[Kra04] I. Krasikov. New bounds on the Hermite polynomials. East J. Approx., 10:1-8, 2004. 2.3

[Li00] D.X. Li. On default correlation: A copula function approach. The Journal of Fixed Income, $9(4): 43-54,2000.1,1$ 
[LK10] O.P. Le Maître and O.M. Knio. Spectral Methods for Uncertainty Quantification. With Applications to Computational Fluid Dynamics. Scientific Computation. Springer, 2010. 2.2

[LMS16] L. Le Gratiet, S. Marelli, and B. Sudret. Metamodel-based sensitivity analysis: polynomial chaos expansions and Gaussian processes. Handbook of Uncertainty Quantification, pages 1-37, 2016. 1

[MFE05] A.J. McNeil, R. Frey, and P. Embrechts. Quantitative risk management. Princeton series in finance. Princeton University Press, 2005. 1

[Nua06] D. Nualart. Malliavin calculus and related topics. Springer Verlag, second edition, 2006. (with corrections on the webpage of the author). 2.2

[San91] G. Sansone. Orthogonal functions. Dover New York, 1991. 2.3, 3.2.2

[Sze75] G. Szego. Orthogonal polynomials, volume 23. American Mathematical Soc., fourth edition, 1975. $2.2,5.2,5.3$

[Usp26] J.V. Uspensky. On the development of arbitrary functions in series of Hermite's and Laguerre's polynomials. Annals of Mathematics, pages 593-619, 1926. 2.3

[Vas91] O. Vasicek. Limiting loan loss probability distribution. KMV corporation, 1991. 1

[YHZ06] J. Yang, T. Hurd, and X. Zhang. Saddlepoint approximation method for pricing CDOs. Journal of Computational Finance, 10(1):1, 2006. 1 